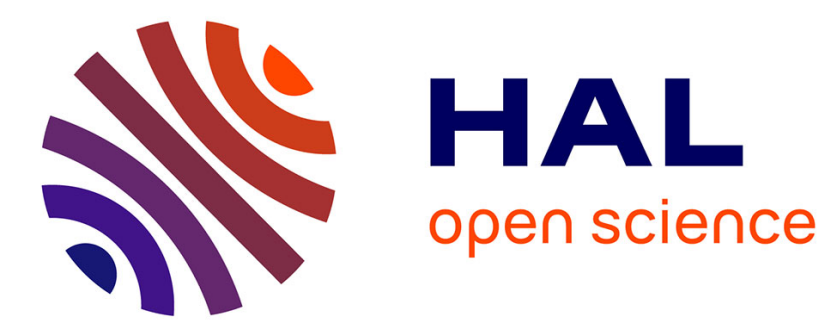

\title{
Non-Local Morphological PDEs and p-Laplacian Equation on Graphs with applications in image processing and machine learning
}

\author{
Abderrahim Elmoataz, Xavier Desquesnes, Olivier Lezoray
}

\section{- To cite this version:}

Abderrahim Elmoataz, Xavier Desquesnes, Olivier Lezoray. Non-Local Morphological PDEs and p-Laplacian Equation on Graphs with applications in image processing and machine learning. IEEE Journal of Selected Topics in Signal Processing, 2012, 6 (7), pp.764-779. 10.1109/JSTSP.2012.2216504 . hal-00813009

\section{HAL Id: hal-00813009 \\ https://hal.science/hal-00813009}

Submitted on 24 Feb 2014

HAL is a multi-disciplinary open access archive for the deposit and dissemination of scientific research documents, whether they are published or not. The documents may come from teaching and research institutions in France or abroad, or from public or private research centers.
L'archive ouverte pluridisciplinaire HAL, est destinée au dépôt et à la diffusion de documents scientifiques de niveau recherche, publiés ou non, émanant des établissements d'enseignement et de recherche français ou étrangers, des laboratoires publics ou privés. 


\title{
Non-Local Morphological PDEs and $p$-Laplacian Equation on Graphs with applications in image processing and machine learning
}

\author{
Abderrahim Elmoataz, Xavier Desquesnes, Olivier Lézoray
}

\begin{abstract}
In this paper, we introduce a new class of nonlocal $p$-Laplacian operators that interpolate between non-local Laplacian and infinity Laplacian. These operators are discrete analogous of the game $p$-laplacian operators on Euclidean spaces, and involve discrete morphological gradient on graphs. We study the Dirichlet problem associated with the new $p$-Laplacian equation and prove existence and uniqueness of it's solution. We also consider non-local diffusion on graphs involving these operators. Finally, we propose to use these operators as a unified framework for solution of many inverse problems in image processing and machine learning.
\end{abstract}

Index Terms $-p$-Laplacian, PDEs-based morphology on graphs, image processing, machine learning, Tug-of-war games.

\section{INTRODUCTION}

\section{A. Motivations and Contributions}

In the last decade, there has been an increasing interest in local and non-local $p$-Laplacian on Euclidean domains and graphs. Indeed, this operator plays an important role in geometry and Partial Differential Equations. Many nonlinear problems in physics, mechanics, image processing or computer vision are formulated in equations that contain the $p$ Laplacian. Non-local continuous version of the $p$-Laplacian appear naturally in the study of many evolution equations as well as in mathematical biology and image processing. For theory and applications see the recent book [1] and reference therein. On the other hand the discrete Laplacian or graph Laplacian has been extensively used in machine learning for clustering, dimension reduction [2]. The graph $p$-Laplacian, generalization of the standard Laplacian has started to attract attention from mathematical, machine learning, image and manifolds processing community. One can quote the proof of the the relationship between graph $p$-Laplacian and cheeger cut [3], the $p$-Laplacian regularization for semi-supervised classification [31], or the recent framework for nonlocal regularization on graphs based on $p$-Laplacian that unify image, mesh and manifold processing [4]. Meanwhile PDEs on graphs with discrete $p$-Laplacian has been investigated as a subject on its own interest dealing with existence and qualitative behavior of the solution [24], [25].

The authors are with the Université de Caen Basse-Normandie and the ENSICAEN in the GREYC Laboratory, Image Team, 6 Boulevard Maréchal Juin, F-14050 CAEN cedex FRANCE.

Email: \{abderrahim.elmoataz-billah, xavier.desquesnes, olivier.lezoray\} @ unicaen.fr

Phone: +33(0)231452706, Fax: +00(0)231452698
In this paper, we introduce a new class of $p$-Laplacian operators involving discrete morphological gradients on graphs. These operators interpolate between non-local Laplacian and infinity Laplacian on graphs and are obtained as the discretization on weighted graphs of the well known normalized $p$-Laplacian or game $p$-laplacian. This adaptation, involving discrete morphological gradients on graphs leads to finite difference equations on graphs with variable coefficients. The study of these equations, under the light of connexions with corresponding continuous PDEs, is a subject of it's own interest. Then, we study the Dirichlet problem associated with these equations and prove existence and uniqueness of solutions. Recently, certain random tug-of-war games have been used in connection with PDEs problems [5], [6], [7]. We then show that value functions of these games coincide with the solution of these $p$-Laplacian equations. And finally, we propose to use these operators as a framework for solving many inverse problems in image processing and machine learning.

So, our first motivation is mainly theoretic and concerns the introduction of a new class of $p$-Laplacian operators on graphs. The second one is more practical and is part of a more general motivation to transpose and extend well-known tools as a framework on weighted graphs.

Indeed, more and more contemporary applications involve data in the form of functions defined on irregular and topologically complicated domains. Typical examples are data defined on manifolds or irregularly-shaped domains, data defined on network-like structures, or data defined as high dimensional point clouds such as collections of features vectors. Such data are not organized as familiar digital signals and images sampled on regular lattices. However, they can be conveniently represented as graphs where each vertex represents measured data and each edge represents a relationship (connectivity or certain affinities or interaction) between two vertices. Processing and analyzing these types of data is a major challenge for both image and machine learning communities. Hence, it is very important to transfer to graphs and networks many of the mathematical tools which were initially developed on usual Euclidean spaces and proven to be efficient for many problems and applications dealing with usual image and signal domains. 


\section{B. Short Background on PDEs on graphs}

Historically, the main tools for the study of graphs or networks come from combinatorial and graph theory. In recent years there has been an increasing interest in the investigation of two major mathematical tools for signal and image analysis, which are PDEs and wavelets, on graphs. For wavelets on graphs, one quote the works of Coifman et al. [8] on diffusion wavelets, Jansen [9] on multiscale methods, or recently Hammond et al. [10] on spectral wavelets transforms. The PDEs approach on graphs starts to raise interests in image and manifold processing, it consists in the exploitation of Partial difference Equations (PdEs), or related Discrete Vector Calculus [11], [12], [13], [14]. Following our previous works, in this paper we only consider PdEs.

Conceptually, PdEs are methods that mimic PDEs on graphs, by replacing differential operators by difference operators on graphs. Historically, one can trace back use of PdEs to the seminal paper by Courant, Friedrichs and Levy [15]. In their paper, the authors introduced the finite difference method as a convenient way of dealing numerically with PDEs. In recent papers, the study of PdEs has appeared to be a subject on its own interest, dealing with existence and qualitative behavior of the solutions [16], [17], [18], [19]. Many discretizations of PDEs on images or surfaces design PdEs restricted to some particular and regular graphs (e.g., grid graphs). For the use of PdEs on graphs of arbitrary topology, with concrete applications in image or manifold processing as well as machine learning, see [4], [14] and references therein. Among these references, one can quote the digitization of the total variation (TV) and the Rudin-Osher-Fatemi (ROF) model of images [20] onto unweighted graphs [21], [22] for image denoising. An extension of this model, that uses a normalized $p$-Dirichlet energy on weighted graphs, is proposed in [23] in the context of semi-supervised learning.

On the roots of these works, we have presented an extension of these models in the context of image, mesh and manifold processing [24], [25], [4], by introducing a nonlocal discrete regularization framework on weighted graphs of arbitrary topology. In particular, it was shown that these regularization processes lead to a family of discrete and semidiscrete diffusion processes based on discrete $p$-Laplacian operators. These processes, parametrized by the graph structure (topology and geometry) and by the degree $p$ of smoothness, allow to perform several filtering tasks such as denoising and simplification. Moreover, local and non-local image regularizations are formalized within the same framework, that corresponds to the transcription of the local or non-local continuous regularizations proposed in [26].

Remark: The term non-local, applied to our discrete operators, is related to the non-locality of data defined on Euclidean domains (as images). Indeed, by graph construction, these operators can mimic non-local operators defined on continuous domain. Then, this term is used as a reference to the continuous case [27] where it means that each element can interact with every other elements in the domain (and not only adjacent ones), and should not be confused with the one in non-local filtering (that uses patches).
With the same idea, by introducing upwind discrete derivatives and gradients, we proposed PdEs morphological processes on graphs that are transcriptions of continuous morphological PDES such as dilation, erosion, leveling or Eikonal equations [28], [29], [30].

\section{Paper Organization}

The rest of the paper will be organized as follows. In Section II, we provide definitions and notations used in this work, and recall our previous works on $p$-Laplacian and PdEsbased morphology on graphs. In Section III, we provide a new interpretation of morphological operators as a family of non-local digital averaging filters, and a new expression of the infinity Laplacian. In Section IV, we present a new family of $p$-Laplacian on graphs, as non-local normalized $p$ Laplacian. Then, we study existence and uniqueness of the Dirichlet problem associated with the $p$-Laplacian equation. A link between Tug-of-War games and this new family is also provided. Section V presents several applications to some interpolation problems in image and high dimensional data. Finally, Section VI concludes the paper.

\section{Definitions And Previous Works}

In this Section, we present definitions and previous works involved in this paper.

\section{A. Partial difference Equations on graphs}

1) Notations: Let us consider the general situation where any discrete domain can be viewed as a weighted graph. A weighted graph $G=(V, E, w)$ consists in a finite set $V$ of $N$ vertices and in a finite set $E \subseteq V \times V$ of edges. Let $(u, v)$ be the edge that connects vertices $u$ and $v$. An undirected graph is weighted if it is associated with a weight function $w: V \times V \rightarrow[0,1]$. The weight function represents a similarity measure between two vertices of the graph. According to the weight function, the set of edges is defined as : $E=$ $\{(u, v) \mid w(u, v) \neq 0\}$. We use the notation $u \sim v$ to denote two adjacent vertices. The degree of a vertex $u$ is defined as $\delta_{w}(u)=\sum_{v \sim u} w(u, v)$. The neighborhood of a vertex $u$ (i.e., the set of vertices adjacent to $u$ ) is denoted $N(u)$. In this paper, the considered graphs are connected, undirected, with no selfloops neither multiple edges. Let $\mathcal{H}(V)$ be the Hilbert space of real valued functions on the vertices of the graph. Each function $f: V \rightarrow \mathbb{R}$ of $\mathcal{H}(V)$ assigns a real value $f(u)$ to each vertex $u \in V$. Similarly, let $\mathcal{H}(E)$ be the Hilbert space of real valued functions defined on the edges of the graph. These two spaces are endowed with following the inner products: $\langle f, h\rangle_{\mathcal{H}(V)}=\sum_{u \in V} f(u) g(u)$ with $f, g \in \mathcal{H}(V)$, and $\langle F, H\rangle_{\mathcal{H}(E)}=\sum_{u \in V} \sum_{v \in V} F(u, v) G(u, v)$ where $F, G \in$ $\mathcal{H}(E)$.

Given a function $f: V \rightarrow \mathbb{R}$, the integral of $f$ is defined as

$$
\int_{V} f=\sum_{u \in V} f(u)
$$


and its $\mathcal{L}_{p}$ norm is given by

$$
\begin{aligned}
\|f\|_{p} & =\left(\sum_{u \in V}|f(u)|^{p}\right)^{1 / p}, 1 \leqslant p<\infty \\
\|f\|_{\infty} & =\max _{u \in V}(|f(u)|), p=\infty
\end{aligned}
$$

Let $\mathcal{A}$ be a set of connected vertices with $\mathcal{A} \subset V$ such that for all $u \in \mathcal{A}$, there exists a vertex $v \in \mathcal{A}$ with $(u, v) \in E$. We denote by $\partial \mathcal{A}$ : the boundary set of $\mathcal{A}$,

$$
\partial \mathcal{A}=\left\{u \in \mathcal{A}^{c}: \exists v \in \mathcal{A} \text { with }(u, v) \in E\right\}
$$

where $\mathcal{A}^{c}=V \backslash \mathcal{A}$ is the complement of $\mathcal{A}$.

2) Differences and gradient operators: We recall several definitions of difference operators on weighted graphs to define derivatives and morphological operators on graphs. More details on these operators can be found in [4], [28].

The non-local gradient or difference operator of a function $f \in \mathcal{H}(V)$, noted $\mathcal{G}_{w}: \mathcal{H}(V) \rightarrow \mathcal{H}(E)$, is defined on an edge $(u, v) \in E$ by:

$$
\left(\mathcal{G}_{w} f\right)(u, v) \stackrel{\text { def. }}{=} \gamma(w(u, v))(f(v)-f(u)) .
$$

where $\gamma: \mathbb{R}^{+} \rightarrow \mathbb{R}^{+}$depends on the weight function (in the sequel we denote $\gamma(w(u, v))$ by $\left.\gamma_{u v}\right)$. This gradient operator is linear and antisymmetric.

The adjoint of the difference operator, noted $\mathcal{G}_{w}^{*}: \mathcal{H}(E) \rightarrow$ $\mathcal{H}(V)$, is a linear operator defined by $\left\langle\mathcal{G}_{w} f, H\right\rangle_{\mathcal{H}(E)}=$ $\left\langle f, \mathcal{G}_{w}^{*} H\right\rangle_{\mathcal{H}(V)}$ for all $f \in \mathcal{H}(V)$ and all $H \in \mathcal{H}(E)$. Using the definitions of the difference and inner products in $\mathcal{H}(V)$ and $\mathcal{H}(E)$, the adjoint operator $\mathcal{G}_{w}^{*}$, of a function $H \in \mathcal{H}(E)$, can by expressed at a vertex $u \in V$ by the following expression:

$$
\left(\mathcal{G}_{w}^{*} H\right)(u) \stackrel{\text { def. }}{=} \sum_{v \sim u} \gamma_{u v}(H(v, u)-H(u, v)) .
$$

The divergence operator, defined by $D_{w}=-\mathcal{G}_{w}^{*}$, measures the net outflow of a function of $\mathcal{H}(E)$ at each vertex of the graph. Each function $H \in \mathcal{H}(E)$ has a null divergence over the entire set of vertices. From previous definitions, it can be easily shown that $\sum_{u \in V} \sum_{v \in V} \mathcal{G}_{w} f(u, v)=0, f \in \mathcal{H}(v)$ and $\sum_{u \in V} D_{w} F(u)=0, F \in \mathcal{H}(E)$.

Based on the previous definitions, we can define two upwind gradients $\mathcal{G}_{w}^{ \pm}: \mathcal{H}(V) \rightarrow \mathcal{H}(E)$, expressed by the following expressions

$$
\mathcal{G}_{w}^{ \pm} f(u, v) \stackrel{\text { def. }}{=} \gamma_{u v}(f(v)-f(u))^{ \pm}
$$

with the notation $(x)^{+}=\max (0, x)$ and $(x)^{-}=-\min (0, x)$.

We define the directional derivative of a function $f \in$ $\mathcal{H}(V)$, noted $\left(\partial_{v} f\right)$ as

$$
\partial_{v} f(u) \stackrel{\text { def. }}{=} \gamma_{u v}(f(v)-f(u))
$$

It is easy to show that the $k$-order directional derivative is

$$
\partial_{v}^{k} f(u)=(-1)^{k} \gamma_{u v}^{k}(f(v)-f(u))
$$

We also introduce two morphological directional partial derivative operators (external and internal), respectively defined as

$$
\left(\partial_{v}^{ \pm} f\right)(u) \stackrel{\text { def. }}{=}\left(\left(\partial_{v} f\right)(u)\right)^{ \pm}
$$

The discrete non-local weighted gradient of a function $f \in$ $\mathcal{H}(V)$, noted $\nabla_{w} f: \mathcal{H}(V) \rightarrow \mathbb{R}^{|\mathrm{V}|}$, is defined on a vertex $u \in V$ as the vector of all partial derivatives with respect to the set of edges $(u, v) \in E$ :

$$
\left(\nabla_{w} f\right)(u) \stackrel{\text { def. }}{=}\left(\left(\partial_{v} f\right)(u)\right)_{v \in V}^{T} .
$$

Similarly, discrete upwind non-local weighted gradients are defined as

$$
\left(\nabla_{w}^{ \pm} f\right)(u) \stackrel{\text { def. }}{=}\left(\left(\partial_{v}^{ \pm} f\right)(u)\right)_{v \in V}^{T} .
$$

The $\mathcal{L}_{p}$ norms, $1 \leqslant p<\infty$ of these gradients : $\left\|\nabla_{w} f\right\|_{p}$ and $\left\|\nabla_{w}^{ \pm} f\right\|_{p}$, allow to define the notion of the regularity of a function around a vertex. They are expressed as :

$$
\left\|\left(\nabla_{w}^{ \pm} f\right)(u)\right\|_{p}=\left[\sum_{v \in V} \gamma_{u v}^{p}(f(v)-f(u))^{ \pm}\right]^{\frac{1}{p}}
$$

Similarly, the $\mathcal{L}_{\infty}$ norm of these gradients is expressed as :

$$
\left\|\left(\nabla_{w}^{ \pm} f\right)(u)\right\|_{\infty}=\max _{v \in V}\left(\gamma_{u v}\left|(f(v)-f(u))^{ \pm}\right|\right)
$$

They can be used to construct several regularization functionals on graphs. One can remark that all these definitions do not depend on the graph structure.

\section{B. Previous works}

In this Section, we first recall our previous works on the expression of the $p$-Laplacian on graphs for $1 \leq p<\infty$ [31], [4] as well as PdEs based mathematical morphology.

1) p-Laplacian: In our previous works, we focused on the expression of the $p$-Laplacian on weighted graphs with $1 \leq p<\infty$. We consider the non-local anisotropic $p$-Laplace operator of a function $f \in \mathcal{H}(V)$, noted $\Delta_{w, p}: \mathcal{H}(V) \rightarrow$ $\mathcal{H}(V)$, defined by:

$$
\left(\Delta_{w, p} f\right)(u)=\frac{1}{2} D_{w}\left(\left|\left(\mathcal{G}_{w} f\right)\right|^{p-2}\left(\mathcal{G}_{w} f\right)\right)(u) .
$$

The non-local anisotropic $p$-Laplace operator of $f \in \mathcal{H}(V)$, at a vertex $u \in V$, can be computed by [32]:

$$
\left(\Delta_{w, p} f\right)(u)=\sum_{v \sim u}\left(\varphi_{w, p} f\right)(u, v)(f(v)-f(u))
$$

with

$$
\left(\varphi_{w, p} f\right)(u, v)=\gamma_{u v}^{p}|f(v)-f(u)|^{p-2} .
$$

This operator is nonlinear if $p \neq 2$. In this latter case, it corresponds to the combinatorial graph Laplacian. To avoid zero denominator in (13) when $p \leq 1,|f(v)-f(u)|$ is replaced by $|f(v)-f(u)|_{\epsilon}=|f(v)-f(u)|+\epsilon$, where $\epsilon \rightarrow 0$ is a small fixed constant. 
In order to simplify the notations, we will now refer to the non local anisotropic $p$-Laplacian as the $p$-Laplacian. Depending on $\gamma_{u v}$ definition, we will consider the two following p-Laplacians:

$$
\begin{gathered}
\gamma_{u v}=\sqrt{w(u, v)} \rightarrow \Delta_{w, p}^{U}: \text { Unnormalized } p \text {-Laplacian } \\
\gamma_{u v}=\sqrt{\frac{w(u, v)}{\delta_{w}(u)}} \rightarrow \Delta_{w, p}: \text { Normalized } p \text {-Laplacian }
\end{gathered}
$$

As an example, in the case where $p=2$, we have :

$$
\begin{array}{r}
\Delta_{w, 2}^{U}=\sum_{v \sim u} w(u, v)(f(v)-f(u)) . \\
\Delta_{w, 2}=\frac{1}{\delta_{w}(u)} \sum_{v \sim u}(w(u, v) f(v))-f(u) .
\end{array}
$$

2) $p$-Laplacian regularization and non-local average filtering: Let $f^{0} \in \mathcal{H}(V)$ be a given function defined on the vertices of a weighted graph $G=(V, E, w)$. In a given context, this function represents an observation of a clean function $h \in \mathcal{H}(V)$ corrupted by an additive noise $n \in \mathcal{H}(V)$ such that $f^{0}=h+n$. To recover the uncorrupted function $h$, the processing task is to remove the noise $n$ from $f^{0}$. A commonly used method is to seek for a function $f \in \mathcal{H}(V)$, which is regular enough on $G$, and also close enough to $f^{0}$. This can be formalized by the minimization of an energy functional which involves a regularization term (or penalty term) plus an approximation one (or fitting term). In this paper, we consider the following model:

$$
\begin{aligned}
& \qquad h \approx \arg \min _{f: V \rightarrow \mathbb{R}} J_{w, p}(f)+\frac{\lambda}{2}\left\|f-f^{0}\right\|_{2}^{2}, \\
& \text { where } \quad J_{w, p}(f) \stackrel{\text { def. }}{=} \frac{1}{2 p} \sum_{u \in V}\left\|\left(\nabla_{w} f\right)(u)\right\|_{p}^{p}
\end{aligned}
$$

is a gradient-based functional, and $\lambda \in \mathbb{R}$ is a regularization parameter, called Lagrange multiplier, that controls the tradeoff between the penalty term and the fitting term.

To get the solution of (20), we consider the following system of equations (Euler-Lagrange equation) [4], [31], :

$$
\frac{\partial J_{w, p}(f)}{\partial f(u)}+\lambda\left(f(u)-f^{0}(u)\right)=0, \quad \forall u \in V,
$$

where the first term denotes the variation of (21) with respect to $f$ at a vertex $u$. It is easy to show that this variation is equal to [4], [31]:

$$
\frac{\partial J_{w, p}(f)}{\partial f(u)}=-\left(\Delta_{w, p} f\right)(u)
$$

Equation (22) can be rewritten as:

$$
\begin{aligned}
\lambda f^{0}(u) & =\left(\lambda+\sum_{v \sim u} \gamma_{u v}^{p}|f(v)-f(u)|^{p-2}\right) f(u) \\
& +\sum_{v \sim u} \gamma_{u v}^{p}|f(v)-f(u)|^{p-2} f(v)
\end{aligned}
$$

Since this is a nonlinear system, an interesting approximate solution is provided by the linearized Gauss-Jacobi iterative algorithm (see [31] for more details), an iteration of which is decomposed in the following two steps:

$$
\begin{cases}\beta_{u v}^{p, n}=\sum_{v \sim u} \gamma_{u v}^{p}\left|f^{n}(v)-f^{n}(u)\right|^{p-2}, & \forall(u, v) \in E, \\ f^{n+1}(u)=\frac{\lambda f^{0}(u)+\sum_{v \sim u} \beta_{u v}^{p, n} f^{n}(v)}{\lambda+\sum_{v \in V} \beta_{u v}^{p, n}}, & \forall u \in V .\end{cases}
$$

This describes a family of neighborhood filters. Indeed, at each iteration, the new value of $f$ at a vertex $u$ depends on two quantities: the initial value $f^{0}(u)$, and a weighted average of the filtered values of $f$ in the neighborhood of $u$. The choice of the regularization parameters and the choice of the graph allow to retrieve and to extend several well-known filters proposed in the context of image smoothing and denoising. In particular, for $p=2$, iteration (25) is rewritten as:

$$
f^{n+1}(u)=\frac{\lambda f^{0}(u)+\sum_{v \sim u} \gamma_{u v}^{2} f^{n}(v)}{\lambda+\sum_{v \sim u} \gamma_{u v}^{2}} .
$$

This filter allows to recover many other filtering schemes, such as bilateral filter, TV-digital filter, non-local means, etc... See [4] for details.

When $\lambda=0$ (no fitting term) and $\gamma_{u v}=\sqrt{w(u, v)}$, we obtain the non-local mean filter : $f^{n+1}(u)=N \operatorname{LM}\left(f^{n}\right)(u)$, with

$$
N L M(f)(u)=\frac{\sum_{v \sim u} w(u, v) f(v)}{\sum_{v \sim u} w(u, v)} .
$$

3) PdE-based morphology on graphs: Adaptive algebraic and PDEs-based morphology have recently received a lot of attention. Such operators can be adaptive with spatially-variant structuring elements or intensity level-adaptive. For instance, one can quote the following works [33], [34], [35] for image processing or [36], [37] that extend the property of translation invariance or morphological operators in a spatially-varying morphology framework. The interested readers can also refer to [38] and references therein for a recent overview of adaptive morphology. A study of mathematical morphology for nonlocal filtering can be found in [39]. In the field of image processing, one also can quote the following works that investigate morphological operators on graphs [40], [41], [42], [43], [44].

Following another approach, in [28], [30], using the expression of weighted morphological gradients (9) based on PdEs, we have defined the discrete analogue of the continuous PDEs-based dilation and erosion formulations. Continuous scale morphology [45] defines flat dilation $\delta$ and erosion $\epsilon$ of a function $f^{0}: \mathbb{R}^{\mathrm{m}} \rightarrow \mathbb{R}$ by using structuring sets $B=\left\{x:\|x\|_{p} \leq 1\right\}$ with the following general PDEs [46]:

$$
\frac{\partial \delta(f)}{\partial t}=\partial_{t} f=+\|\nabla f\|_{p} \text { and } \frac{\partial \varepsilon(f)}{\partial t}=\partial_{t} f=-\|\nabla f\|_{p},
$$

where $f$ is a modified version of $f^{0}, \nabla$ is the gradient operator, $\|\cdot\|_{p}$ corresponds to the $\mathcal{L}_{p}$-norm, and one has the initial condition $f=f^{0}$ at $t=0$. We have proposed in [28] the discrete PdEs analogue of PDEs-based dilation and erosion formulations and obtained the following expressions 
over graphs. For a given initial function $f^{0} \in \mathcal{H}(V)$ :

$$
\begin{aligned}
& \frac{\partial \delta(f)(u)}{\partial t}=\partial_{t} f(u)=+\left\|\left(\nabla_{w}^{+} f\right)(u)\right\|_{p}, \text { and } \\
& \frac{\partial \varepsilon(f)(u)}{\partial t}=\partial_{t} f(u)=-\left\|\left(\nabla_{w}^{-} f\right)(u)\right\|_{p} \quad, \forall u \in V
\end{aligned}
$$

where $\nabla_{w}^{+}$and $\nabla_{w}^{-}$are weighted pseudo-morphological gradients (9). (We use the term morphological by analogy with the continuous case) The relation between this morphological framework and adaptive methods proposed in the literature such as amoebas [39] or PDEs-based viscous morphology [47] has been discussed in [30]. In the sequel, we will show that for particular values of $p(1, \infty)$, resolutions of these equations can be interpreted as average filters. For the sake of clarity, we will simply call them Non Local Dilation (NLD) and Non Local Erosion (NLE), instead of Non Local Pseudo Dilation (respectively Erosion).

\section{FROM PDES-BASED MORPHOLOGY TO AVERAGE FILTERING OPERATORS AND INFINITY LAPLACIAN}

Before presenting our new formulation of non-local $p$ Laplacian, we will first introduce some new expressions and results involved in this formulation. First, we will provide new interpretation of morphological operators as a family of non-local digital averaging filters. Then, we will present a new expression of the $\infty$-Laplacian, based on morphological gradients, and show that this new expression is linked with the newly introduced family of non-local digital filters.

\section{A. Non-local averaging filters}

In this Section, we will show that morphological operators (29) can be interpreted as a family of non-local digital averaging filters, according to the value of $p$ and the graph topology.

- Case $p=\infty$ : The dilation process (29) can be expressed as the following iterative PdE:

$$
\begin{aligned}
f^{n+1}(u) & =f^{n}(u) \\
& +\Delta t \max _{u \sim v}\left(\sqrt{w(u, v)} \max \left(f^{n}(v)-f^{n}(u), 0\right)\right) .
\end{aligned}
$$

In the case where $\Delta t=1$, this previous PdE can be interpreted as an iterative non-local dilation process $f^{n+1}=N L D\left(f^{n}\right)$ where $N L D: \mathcal{H}(V) \rightarrow \mathcal{H}(V)$ is defined according to (30).

In the case where $w=1$, and with a particular graph construction $(u \in N(u))$ the NLD recovers the true morphological formulation, as :

$$
\begin{aligned}
f^{n+1}(u) & =f^{n}(u)+\max _{v \sim u}\left(\max \left(f^{n}(v)-f^{n}(u), 0\right)\right) \\
& =f^{n}(u)+\max _{v \sim u}\left(f^{n}(v)-f^{n}(u)\right) \\
& =\max _{v \sim u}\left(f^{n}(v)\right)
\end{aligned}
$$

In a more general case, with an arbitrary weight $w$, equation (30) can be rewritten as

$f^{n+1}(u)=\left(1-\sqrt{w\left(u, v_{m}\right)}\right) f^{n}(u)+\sqrt{w\left(u, v_{m}\right)} f^{n}\left(v_{m}\right)$,

where $v_{m}=\arg \min _{v \sim u}(f(v)-f(u))$.

The non-local erosion NLE is defined similarly and can be interpreted as a minimum between $f(u)$ and every $f(v)$ such that $v$ is similar and in the neighborhood of $u$.

- Case $p=1$ :

$$
\begin{aligned}
f^{n+1}(u) & =f^{n}(u) \\
& +\Delta t \sum_{v \sim u} w(u, v) \max \left(f^{n}(v)-f^{n}(u), 0\right)
\end{aligned}
$$

can be rewritten as

$$
\begin{aligned}
f^{n+1}(u) & =f^{n}(u)+\Delta t \sum_{v \in V_{u}^{+n}} w(u, v)\left(f^{n}(v)-f^{n}(u)\right) \\
f^{n+1}(u) & =f^{n}(u)\left(1-\Delta t \sum_{v \in V_{u}^{+n}} w(u, v)\right) \\
& +\Delta t \sum_{v \in V_{u}^{+n}} w(u, v) f^{n}(v)
\end{aligned}
$$

where $V_{u}^{+n}=\left\{v \sim u \mid f^{n}(v)>f^{n}(u)\right\}$. Now, if we define $\Delta t$ as

$$
\Delta t=\max _{u \in V}\left(\frac{1}{\sum_{v \in V_{u}^{+n}} w(u, v)}\right),
$$

the dilation process can be expressed with

$$
\begin{cases}f^{n+1}(u)=\frac{\sum_{v \in V_{u}^{+n}} w(u, v) f^{n}(v)}{\sum_{v \in V_{u}^{+n}} w(u, v)} & \text { if } V_{u}^{+n} \neq \emptyset \\ f^{n+1}(u)=f^{n}(u) & \text { otherwise }\end{cases}
$$

which is a non-local average filtering.

Similarly the erosion process can be expressed as

$$
\begin{cases}f^{n+1}(u)=\frac{\sum_{v \in V_{u}^{-n}} w(u, v) f^{n}(v)}{\sum_{v \in V_{u}^{-n}} w(u, v)} & \text { if } V_{u}^{-n} \neq \emptyset \\ f^{n+1}(u)=f^{n}(u) & \text { otherwise }\end{cases}
$$

where $V_{u}^{-n}=\left\{v \sim u \mid f^{n}(v)<f^{n}(u)\right\}$.

Finally, these two PDEs, (35) and (36), define a new class of conditional non-local means expressed as conditional non-local dilation, and erosion. These operators can be interpreted as non-local means, where the neighborhood used to compute the mean is restricted to the subset of neighbors the value of $f$ is superior (respectively inferior) to $f(u)$. In the sequel, we denote them $N L M^{+}$ (Non-Local Mean Dilation) and respectively $N L M^{-}$ (Non-Local Mean Erosion).

- Case $p=2$, with a constant weight function $(w=1)$. We have shown in a previous paper [28] that these discrete 
morphological operators recover the exact Osher-Sethian first order upwind discretization schemes for PDEs based morphology [48].

\section{B. anisotropic p-Laplacian as a morphological process}

We now provide a new relationship between the anisotropic Laplacian and morphological gradients. We show this new relation in the sequel.

Proposition 1: For $1 \leq p<+\infty$, at a vertex $u \in V$

$$
\left(\Delta_{w,(p+1)} f\right)(u)=\left\|\left(\nabla_{w^{\prime}}^{+} f\right)(u)\right\|_{p}^{p}-\left\|\left(\nabla_{w^{\prime}}^{-} f\right)(u)\right\|_{p}^{p}
$$

with $w^{\prime}(u, v)=w(u, v)^{\frac{p+1}{p}}$.

Proof: From (13) and (10), we have

$$
\left(\Delta_{w, p} f\right)(u)=\sum_{v \sim u} w_{u v}^{p / 2}|f(v)-f(u)|^{p-2}(f(v)-f(u)) .
$$

Since $|x|=x^{+}+x^{-}$and $x=x^{+}-x^{-}$, one has, with $A=$ $(f(v)-f(u))$ :

$$
\left(\Delta_{w,(p+1)} f\right)(u)=\sum_{v \sim u} w_{u v}^{\frac{p+1}{2}}\left(A^{-}-A^{+}\right)\left(A^{+}+A^{-}\right)^{p-1} .
$$

Then, by developing $\left(A^{+}+A^{-}\right)^{p-1}$, since $A^{+} A^{-}=0$, it is easy to obtain (37).

If we take some particular values of $p$, we have the following relations. For $p=2$, (37) gives

$$
\left(\Delta_{w, 2} f\right)(u)=\left\|\left(\nabla_{w^{2}}^{+} f\right)(u)\right\|_{1}-\left\|\left(\nabla_{w^{2}}^{-} f\right)(u)\right\|_{1} .
$$

The classical combinatorial Laplacian operator can therefore be expressed as the difference of two morphological external and internal gradients. This new expression of the $p$-Laplacian is the basis of our proposition for an expression of the infinity Laplacian on graphs.

\section{The infinity Laplacian on graphs}

The infinity Laplacian operator is related to the PDE infinity Laplacian equation that can be expressed as

$$
-\Delta_{\infty} f=0
$$

where the expression

$$
\Delta_{\infty} f=\sum_{i, j}^{n} \frac{\partial f}{\partial_{x_{i}}} \frac{\partial f}{\partial_{x_{j}}} \frac{\partial f}{\partial_{x_{i}} \partial_{x_{j}}}
$$

denotes the infinity Laplacian on Euclidean domains for smooth functions in some open sets $\Omega \subset \mathbb{R}^{\mathrm{n}}$. Aronsson [49] interpreted formally $\Delta_{\infty} f=0$ as the limit of the minimization problem of $\int\|\nabla f\|^{p} d x$ as $p \rightarrow \infty$, under given boundary conditions.

As in the continuous case, this operator can be formally derived as minimization of the following energy on graphs, as $p$ goes to the infinity.

$$
J_{w, p}(f)=\sum_{u \in V}\left\|\nabla_{w} f(u)\right\|_{p}^{p}
$$

This minimization leads to the $p$-Laplacian equation $\Delta_{w, p} f(u)=0, \forall u \in V$.

From proposition 1 , we have

$$
\left(\Delta_{w, p} f\right)(u)=\left\|\left(\nabla_{w^{\prime}}^{+} f\right)(u)\right\|_{p-1}^{p-1}-\left\|\left(\nabla_{w^{\prime}}^{-} f\right)(u)\right\|_{p-1}^{p-1}=0
$$

with $w^{\prime}(u, v)=w(u, v)^{\frac{p}{p-1}}$ that we can easily simplify into

$$
\left\|\left(\nabla_{w^{\prime}}^{+} f\right)(u)\right\|_{p-1}-\left\|\left(\nabla_{w^{\prime}}^{-} f\right)(u)\right\|_{p-1}=0 .
$$

Using (43) and letting $p \rightarrow \infty$, one can have formally

$$
\left\|\left(\nabla_{w}^{+}\right) f(u)\right\|_{\infty}-\left\|\left(\nabla_{w}^{-} f\right)(u)\right\|_{\infty}=0 .
$$

Based on proposition 1, and the previous limit, we propose a new definition of the $\infty$-Laplacian:

$$
\left(\Delta_{w, \infty} f\right)(u) \stackrel{\text { def. }}{=} \frac{1}{2}\left[\left\|\left(\nabla_{w}^{+} f\right)(u)\right\|_{\infty}-\left\|\left(\nabla_{w}^{-} f\right)(u)\right\|_{\infty}\right]
$$

With an appropriate weight function $(w(u, v)=1$, for all $(u, v) \in E)$, the proposed definition recovers well-known finite difference approximation for the infinity Laplacian. Indeed, (45) can be rewritten as (with $u \sim u$ ):

$$
\left(\Delta_{1, \infty} f\right)(u)=\frac{1}{2}\left[\max _{v \sim u} f(v)+\min _{v \sim u} f(v)\right]-f(u)
$$

For instance $\left(\Delta_{1, \infty} f\right)(u)$ is known in the community of (algebraic) mathematical morphology as the morphological Laplacian. For a general weighted graph, equation (45) can be rewritten as a morphological operator on graphs, involving non-local dilation and non-local erosion previously defined.

$$
\begin{aligned}
& \left(\Delta_{w, \infty} f\right)(u) \\
& =\frac{1}{2}\left[2 f(u)+\left\|\left(\nabla_{w}^{+} f\right)(u)\right\|_{\infty}-\left\|\left(\nabla_{w}^{-} f\right)(u)\right\|_{\infty}\right]-f(u) \\
& =\frac{1}{2}[N L D f(u)+N L E f(u)]-f(u)
\end{aligned}
$$

\section{NON-LOCAL NORMALIZED $p$-LAPLACIAN}

Considering our framework of PdEs, with the discretization of $p$-Laplacian and infinity Laplacian on a general graph domain, we now propose a new family of $p$-Laplacian obtained by the discretization of the game $p$-Laplacian.

\section{A. Definition}

We recall that the $p$-Laplacian of a function $f: \Omega \subset \mathbb{R}^{\mathrm{n}} \rightarrow$ $\mathbb{R}$ is given as

$$
\Delta_{p} f=\operatorname{div}\left(|\nabla f|^{p-2} \cdot \nabla f\right)=|\nabla f|^{p-2}\left\{(p-2) \Delta_{\infty} f+\Delta f\right\} .
$$

The normalized version of this $p$-laplacian operator, referred to game $p$-laplacian is defined [50] as

$$
\Delta_{p}^{N} f(u)=\frac{1}{p|\nabla f|^{p-2}} \Delta_{p} f
$$

This operator was recently introduced to model a stochastic game with noise called Tug of war game. We can write that

$$
\begin{aligned}
\Delta_{p}^{N} f & =\frac{(p-2)}{p} \Delta_{\infty}^{N} f+\frac{1}{p} \Delta f \\
& =\alpha \Delta_{\infty}^{N} f+\beta \Delta f
\end{aligned}
$$


with $\alpha=(p-2) / p$ and $\beta=1 / p$ and where

$$
\Delta_{\infty}^{N} f=\frac{1}{|\nabla f|^{2}} \Delta_{\infty} f
$$

With a simple discretization of (49) and using discrete version of $p$-Laplacian, we propose a non-local version of the normalized $p$-Laplacian. This is given by the following equation.

$$
\Delta_{w, \alpha, \beta} f(u) \stackrel{\text { def }}{=} \alpha\left(\Delta_{w, \infty} f\right)(u)+\beta\left(\Delta_{w, 2} f\right)(u) .
$$

Usual Laplacians and infinity Laplacian can be easily recovered with particular values of $\alpha$ and $\beta$, as:

$$
\begin{aligned}
\Delta_{w, 1,0} & =\Delta_{w, \infty} \\
\Delta_{w, 0,1} & =\Delta_{w, 2}
\end{aligned}
$$

Using (46), equation (51) can be rewritten as a morphological operator involving non-local erosion and non-local dilation

$$
\Delta_{w, \alpha, \beta} f=\frac{\alpha}{2}[N L D(f)+N L E(f)]+\beta N L M(f)-f
$$

Connection with non-local PdEs : Similarly, given a Euclidean graph $G(V, E, w)$, with $V=\Omega \subset \mathbb{R}^{\mathrm{n}}, E=\{(x, y) \in$ $V \times V \mid w(x, y)>0\}$ and

$$
w(x, y)= \begin{cases}\frac{1}{|x-y|^{2 s}} & x \neq y, s \in[0,1] \\ 0 & \text { otherwise. }\end{cases}
$$

In the case where $\beta=0$, our formulation (53) corresponds to recently proposed Hölder infinity Laplacian equation proposed by Chambolle et al. in [51].

$$
\begin{aligned}
\Delta_{w, \infty} f(x)=\frac{1}{2} & {\left[\max _{y \in \Omega, y \neq x}\left(\frac{f(y)-f(x)}{|y-x|^{s}}\right)\right.} \\
& \left.+\min _{y \in \Omega, y \neq x}\left(\frac{f(y)-f(x)}{|y-x|^{s}}\right)\right]
\end{aligned}
$$

This operator is formally derived from the minimization of an energy on the form

$$
\int_{\Omega} \int_{\Omega} \frac{\left[f(y)-\left.f(x)\right|^{p}\right.}{|x-y|^{p \times s}} d x d y
$$

as $p$ goes to infinity. In [51], it was proved that the following equation

$$
\begin{cases}\Delta_{w, \infty} f(u)=0, & u \in \Omega \\ f(u)=g(u), & u \in \partial \Omega\end{cases}
$$

(and with some conditions on $g$ ), has a unique solution.

In the case where $\alpha=0$, this formulation recovers the nonlocal $p$-Laplacian, as

$$
\frac{1}{\delta_{w}(x)} \int_{\Omega} w(x, y)(f(y)-f(x)) d y .
$$

Finally, in the most general case (with any $\alpha, \beta$ and $w$ ), equation (53) corresponds to a PDE which interpolates between infinity Laplacian and Laplacian.
B. Study of the existence and uniqueness of the p-Laplacian equation

Now, we will study the Dirichlet problem associated to this non-local $p$-Laplacian equation (51).

Proposition 2: Given a connected graph $G=(V, E, w)$, a set $A \subset V$ and a function $g: \partial A \rightarrow \mathbb{R}$ where $\partial A$ is the boundary of $A$. Then, there exists a unique function $f \in$ $\mathcal{H}(V)$ such that $f$ verifies the following equation:

$$
\begin{cases}\alpha\left(\Delta_{w, \infty} f\right)(u)+\beta\left(\Delta_{w, 2} f\right)(u)=0 & u \in A \\ f(u)=g(u) & u \in \partial A\end{cases}
$$

with $\alpha, \beta \in \mathbb{R}^{+}$and $\alpha+\beta=1$.

For a proof of existence and uniqueness, we introduce the following non-local average operator (NLA)

$$
N L A(f)=\frac{\alpha}{2}[N L D(f)+N L E(f)]+\beta N L M(f)
$$

and equation (59) can be rewritten as $f(u)=N L A(f)(u)$.

First, let us prove the uniqueness of the solution by using the comparison principle.

Proof: Given two functions $f$ and $h$, we will prove that if $f=N L A(f)$ and $h=N L A(h)$ with $f \leq h$ on $\partial A$, then, $f \leq h$ on the whole domain $V$. By the argument of contradiction, we assume that there exists $M$ such that

$$
M=\sup _{V}(f-h)>0 .
$$

Let $B=\{u \in A: f(u)-h(u)=M\}$. By construction we have $B \neq \emptyset$ and $B \cap \partial A=\emptyset$. We claim that there exists $u_{0} \in B$ and $v \in N\left(u_{0}\right)$, such that $v \notin B$. Otherwise, if for each $u \in A$ and for each $v \in N(u)$ we have $v \notin B$, then it implies that $B \cap \partial A \neq \emptyset$, since the graph is connected : there is a contradiction. Then, from the definition of $M$, we have

$$
\begin{array}{ll}
f\left(u_{0}\right)-h\left(u_{0}\right) \geq f(u)-h(u) & \forall u \in N\left(u_{0}\right) \\
h(u)-h\left(u_{0}\right) \geq f(u)-f\left(u_{0}\right) & \forall u \in N\left(u_{0}\right) .
\end{array}
$$

In particular we can write,

$$
h(v)-h\left(u_{0}\right)>f(v)-f\left(u_{0}\right) .
$$

From these inequalities, and using definitions of non local morphological processes, we have

$$
\begin{gathered}
\max _{u \sim u_{0}}\left(\sqrt{w\left(u_{0}, u\right)} \max \left(h(u)-h\left(u_{0}\right), 0\right)\right) \\
\geq \\
\max _{u \sim u_{0}}\left(\sqrt{w\left(u_{0}, u\right)} \max \left(f(u)-f\left(u_{0}\right), 0\right)\right) \\
N L D(h)\left(u_{0}\right)-h\left(u_{0}\right) \geq N L D(f)\left(u_{0}\right)-f\left(u_{0}\right)
\end{gathered}
$$

similarly

$$
\begin{gathered}
\max _{u \sim u_{0}}\left(\sqrt{w\left(u_{0}, u\right)} \min \left(h(u)-h\left(u_{0}\right), 0\right)\right) \\
\geq \\
\max _{u \sim u_{0}}\left(\sqrt{w\left(u_{0}, u\right)} \min \left(f(u)-f\left(u_{0}\right), 0\right)\right) \\
h\left(u_{0}\right)-N L E(h)\left(u_{0}\right) \geq f\left(u_{0}\right)-N L E(f)\left(u_{0}\right)
\end{gathered}
$$


and finally.

$$
\begin{gathered}
\frac{\sum_{u \sim u_{0}} w\left(u_{0}, u\right)\left(h(u)-h\left(u_{0}\right)\right)}{\sum_{u \sim u_{0}} w\left(u_{0}, u\right)} \\
>\quad \sum_{u \sim u_{0}} w\left(u_{0}, u\right)\left(f(u)-f\left(u_{0}\right)\right) \\
\sum_{u \sim u_{0}} w\left(u_{0}, u\right) \\
N L M(h)\left(u_{0}\right)-h\left(u_{0}\right)>N L M(f)\left(u_{0}\right)-f\left(u_{0}\right)
\end{gathered}
$$

This previous inequality is strict because we know there is $v \in N\left(u_{0}\right)$ such that $h(v)-h\left(u_{0}\right)>f(v)-f\left(u_{0}\right)$.

From relations (61), (62) and (63), we can write the following inequality

$$
\begin{aligned}
& \frac{\alpha}{2}\left[N L D(h)\left(u_{0}\right)+N L E(h)\left(u_{0}\right)\right]+\beta N L M(h)\left(u_{0}\right)-h\left(u_{0}\right) . \\
& > \\
& \frac{\alpha}{2}\left[N L D(f)\left(u_{0}\right)+N L E(f)\left(u_{0}\right)\right]+\beta N L M(f)\left(u_{0}\right)-f\left(u_{0}\right) .
\end{aligned}
$$

and

$$
\begin{aligned}
N L A(h)\left(u_{0}\right)-h\left(u_{0}\right) & >N L A(f)\left(u_{0}\right)-f\left(u_{0}\right) \\
h\left(u_{0}\right)-h\left(u_{0}\right) & >f\left(u_{0}\right)-f\left(u_{0}\right) \\
0 & >0
\end{aligned}
$$

This shows a contradiction and concludes the proof.

Now, let us prove existence.

Proof: First, we recall the Bouwer fixed point theorem : A continuous function from a convex, compact subset of an Euclidean space to itself has a fixed point.

Then, we identify $\mathcal{H}(V)$ as $\mathbb{R}^{\mathrm{n}}$ and consider the set $K=$ $\{f \in \mathcal{H}(V) \mid f(u)=g(u) \forall u \in \partial A$, and $m \leqslant f(u) \leqslant$ $M \forall u \in A\}$, where $m=\min _{\partial A}(g(u))$ and $m=\max _{\partial A}(g(u))$. By definition, $K$ is a convex and compact subset of $\mathbb{R}^{\mathrm{n}}$.

It is easy to show that the map $f \rightarrow N L A(f)$ is continuous and take from $K$ to $K$. So, by the Bouwer fixed point theorem, the map $N L A$ has a fixed point that is solution of $N L A(f)=$ $f$. This complete the proof.

\section{From p-Laplacian on graphs to Tug-of-War games}

In this Section, we show that the value functions of Tug-of-War (TOW) games coincide with the solution of the Dirichlet problem (59) on particular weighted graphs.

Tug-of-War game: The tug-of-war games related to the infinity Laplacian studied in [5] can be briefly described as follows: a tug-of-war game is a two-person, zero-sum game, where two players are in contest and the total earnings of one are the losses of the other. The rules of the game are the following: consider a bounded domain $\Omega \subset \mathbb{R}^{\mathrm{n}}$ and $g: \partial \Omega \rightarrow$ $\mathbb{R}$ a Lipschitz continuous function (the final payoff function). At the initial time, a token is placed at a point $x_{0} \in \Omega$. Then a (fair) coin is tossed and the winner of the toss is allowed to move the game position to any $x_{1} \in B_{\varepsilon}\left(x_{0}\right)$ (where $B_{\varepsilon}(x)=$ $\{y|| y-x \mid \leqslant \varepsilon\}$. At each turn, the coin is tossed again, and the winner chooses a new game state $x_{k} \in B_{\varepsilon}\left(x_{k-1}\right)$. Once the token has reached some $x_{\tau} \in \partial \Omega$, the game ends and the first player earns $g\left(x_{\tau}\right)$ (while the second player earns $-g\left(x_{\tau}\right)$ ). The values of the game for player I and II, $f_{I}\left(x_{0}\right), f_{I I}\left(x_{0}\right)$, are the least possible outcomes that each of the players expect to get when the $\varepsilon$-game starts as $x_{0}$. In [7] it has been shown that this game has an expected value $f_{\varepsilon}\left(x_{0}\right)$ (called the value of the game) that verifies the Dynamic Programming Principle (DPP),

$$
f_{\varepsilon}(x)=\frac{1}{2} \max _{y \in B_{\varepsilon}(x)} f_{\varepsilon}(y)+\frac{1}{2} \min _{y \in B_{\varepsilon}(x)} f_{\varepsilon}(y), \forall x \in \Omega,
$$

here it is understood that $f_{\varepsilon}(x)=g(x)$ for $x \in \partial \Omega$. This formula can be intuitively explained from the fact that the first player tries to maximize the expected outcome (and has the probability $1 / 2$ of selecting the next state of the game) while the second tries to minimize the expected outcome (and also has the probability $1 / 2$ of choosing the next position). The authors also prove that the $\varepsilon$-value of the game is a Lipschitz function converging uniformly as $\varepsilon$ goes to zero, named the continuous value of the game, which solves the infinity Laplacian Equation (50).

Tug-of-War game with noise: If the game is modified as follows: at point $x$ in $\Omega$, player I and player II play $\epsilon$-step tugof-war game with probability $\alpha$, and with probability $\beta$ such that $\alpha+\beta=1$, a random point in ball of radius $\epsilon$ centered at $x$ is chosen. The value functions of the game satisfy the Dynamic Programming Principle

$$
\begin{aligned}
f(x) & =\frac{\alpha}{2}\left[\max _{y \in B_{\epsilon}(x)} f(y)+\min _{y \in B_{\epsilon}(x)} f(y)\right] \\
& +\frac{\beta}{\left|B_{\epsilon}(x)\right|} \int_{B_{\epsilon}(x)} f(y) d y
\end{aligned}
$$

with $f(x)=g(x)$ for $x$ in $\Omega$ where $\alpha$ and $\beta \in \mathbb{R}^{+}$such as $\alpha+\beta=1$. A detail proof for existence and uniqueness of theses type of functions was shown in [7].

In the general case, it was proved in [6] that functions that satisfy (65) with $\beta \neq 0$ approximate solutions of the $p$-Laplacian equation $\Delta_{p} f=\operatorname{div}\left(|\nabla f|^{p-2} . \nabla f\right)=0$.

Let us rewrite (65) in the context of our PdEs framework. We consider the following Euclidean $\varepsilon$-adjacency graph $G(V, E, w)$ with $V=\Omega \subset \mathbb{R}^{\mathrm{n}}, E=\{(x, y) \in \Omega \times$ $\Omega \mid w(x, y)>0\}$. and

$$
w(x, y)= \begin{cases}1 & |y-x| \leqslant \varepsilon \\ 0 & \text { otherwise }\end{cases}
$$

For this particular case, and for more clarity, the weighted gradient and $p$-Laplacian are denoted as $\nabla_{1}$, respectively $\Delta_{1, p}$. Using the definitions of the $\mathcal{L}_{\infty}$ norms of morphological gradients (11), it is easy to obtain the following relations.

$$
\begin{aligned}
& \max _{B_{\varepsilon}(x)} f(y)=\left\|\left(\nabla_{1}^{+} f\right)(x)\right\|_{\infty}+f(x) \\
& \min _{B_{\varepsilon}(x)} f(y)=f(x)-\left\|\left(\nabla_{1}^{-} f\right)(x)\right\|_{\infty} .
\end{aligned}
$$


In the case where $\beta=0$ and $\alpha=1$, and using the previous morphological operators, one can transpose (65) as

$$
\begin{aligned}
f(u) & =\frac{1}{2}\left[\max _{v \sim u} f(v)+\min _{v \sim u} f(v)\right], \\
& =\frac{1}{2}\left[2 f(u)+\left\|\left(\nabla_{1}^{+} f\right)(u)\right\|_{\infty}-\left\|\left(\nabla_{1}^{-} f\right)(u)\right\|_{\infty}\right] \\
& \Leftrightarrow \frac{1}{2}\left[\left\|\left(\nabla_{1}^{+} f\right)(u)\right\|_{\infty}-\left\|\left(\nabla_{1}^{-} f\right)(u)\right\|_{\infty}\right]=0
\end{aligned}
$$

Finally, in the case where $\beta \neq 0$, we obtain the following transposition of DPP (65)

$$
\begin{aligned}
f(u) & =\frac{\alpha}{2}\left[2 f(u)+\left\|\left(\nabla_{1}^{+} f\right)(u)\right\|_{\infty}-\left\|\left(\nabla_{1}^{-} f\right)(u)\right\|_{\infty}\right] \\
& +\frac{\beta}{N(u)} \int_{v \in N(u)} f(v) d v
\end{aligned}
$$

Then, using the formulation of the non-local infinity Laplacian (45) on graphs, equation (68) can be rewritten as

$$
f(u)=\frac{\alpha}{2}\left[2 f(u)+\left(\Delta_{1, \infty} f\right)(u)\right]+\frac{\beta}{N(u)} \int_{v \in N(u)} f(v) d v .
$$

Similarly, the second term of the previous equation can be rewritten using the formulation of the normalized Laplacian on graphs (16).

$$
\frac{1}{N(u)} \int_{v \in N(u)} f(v) d v=\left(f(u)-\left(\Delta_{1,2} f\right)(u)\right)
$$

and then,

$$
f(u)=\frac{\alpha}{2}\left(2 f(u)-\left(\Delta_{1, \infty} f\right)(u)\right)+\beta\left(f(u)-\left(\Delta_{1,2} f\right)(u)\right),
$$

which corresponds to our newly introduced definition of the non-local $p$-Laplacian on graphs.

\section{D. p-Laplacian diffusion on graphs}

In image processing, non-local models have shown their great advantages overall classical local models, since smoothness is not required. They have also shown their ability to preserve geometric and repetitive structures in images (as textures). In this Section, we study a non-local diffusion problem involving the non-local $p$-Laplacian operator and we will show that the corresponding $p$-Laplacian diffusion process can be written as an iterative non-local average filtering process. Given the following equation

$$
\begin{cases}\frac{\partial f(u, t)}{\partial t} & =\Delta_{w, \alpha, \beta} f(u, t) \\ f(u, t=0) & =f^{0}(u)\end{cases}
$$

where $f: V \times[0, T] \rightarrow \mathbb{R}$ is a function and $f^{0}: V \rightarrow \mathbb{R}$ the initial value of $f$. One has

$$
\frac{\partial f^{(u, t)}}{\partial t}=\frac{f^{n+1}(u)-f^{n}(u)}{\Delta t}
$$

where $f^{n}(u)=f(u, n \Delta t), \Delta t=\lambda$.
Then,

$$
\begin{aligned}
f^{n+1}(u)= & f^{n}(u)+\lambda \alpha \Delta_{w, \infty} f^{n}(u)+\lambda \beta \Delta_{w, 2} f^{n}(u) . \\
= & f^{n}(u)+\lambda \alpha\left[\frac{\left\|\left(\nabla_{w}^{+} f\right)(u)\right\|_{\infty}-\left\|\left(\nabla_{w}^{-} f\right)(u)\right\|_{\infty}}{2}\right] \\
& +\lambda \beta\left[\frac{\sum w(u, v) f^{n}(u)}{\delta_{w}(u)}-f^{n}(u)\right] . \\
= & (1-\lambda) f^{n}(u) \\
& +\lambda \alpha\left[\frac{N L E\left(f^{n}\right)(u)+N L D\left(f^{n}\right)(u)}{2}\right] \\
& +\lambda \beta N \operatorname{LM}\left(f^{n}\right)(u) .
\end{aligned}
$$

One can remark that in the case where $\lambda=1$, equation (74) can be rewritten as a non local average:

$$
f^{n+1}(u)=N L A\left(f^{n}\right)(u)
$$

One can also remark that such process is a digital filtering process that combines non-local morphology (erosion and dilation) and non-local means.

\section{APPLICATIONS TO INVERSE PROBLEMS ON WEIGHTED GRAPHS}

In this Section, we illustrate the behavior of normalized $p$ Laplacian and morphological operators presented in this paper, through some inverse problems as functions restoration or interpolation on graphs. The experiments provided are not here to solve a particular application but to illustrate the potentialities of our proposal. Moreover, our aim is not to compare the benefits of $p$-Laplacian versus mathematical morphology for the inverse problems we consider. As a consequence, we will not compare both methods but show the benefits of each method as a sole methodology. In addition, since the processes we propose on graphs are extensions of classical methods when one considers specific graph topologies and graph weights, we will not provide comparison for each applications and refer the interested reader to our other papers that show the benefits of our approaches over conventional methods.

\section{A. Graph construction}

There exists several popular methods to transform discrete data $\left\{x_{1}, \ldots x_{n}\right\}$ into a weighted graph structure. Considering a set of vertices $V$ such that data are embedded by functions of $\mathcal{H}(V)$, the construction of such graph consists in modeling the neighborhood relationships between the data through the definition of a set of edges $E$ and using a pairwise distance measure $\mu: V \times V \rightarrow \mathbb{R}^{+}$. In the particular case of images, the ones based on geometric neighborhoods are particularly well-adapted to represent the geometry of the space, as well as the geometry of the function defined on that space. One can quotes:

- Grid graphs which are most natural structures to describe an image with a graph. Each pixel is connected by an edge to its adjacent pixels. Classical grid graphs are 4-adjacency grid graphs and 8-adjacency grid graphs. Larger adjacency can be used to obtain non-local graphs. 
- Region adjacency graphs (RAG) which provide very useful and common ways of describing the structure of a picture: vertices represent regions and edges represent region adjacency relationship.

- $k$-neighborhood graphs ( $k$-NNG) where each vertex $v_{i}$ is connected with it(s $k$-nearest neighbors according to $\mu$. Such construction implies to build a directed graph, as the neighborhood relationship is not symmetric. Nevertheless, an undirected graph can be obtained while adding an edge between two vertices $v_{i}$ and $v_{j}$ if $v_{i}$ is among the $k$-nearest neighbor of $v_{j}$ or if $v_{j}$ is among the $k$-nearest neighbor of $v_{i}$

- $k$-Extended RAG ( $k$-ERAG) which are RAGs extended by a $k$-NNG. Each vertex is connected to adjacent regions vertices and to it's $k$ most similar vertices of $V$.

The similarity between two vertices is computed according to a measure of similarity $g: E \rightarrow \mathbb{R}^{+}$, which satisfies:

$$
w(u, v)= \begin{cases}g(u, v) & \text { if }(u, v) \in E \\ 0 & \text { otherwise }\end{cases}
$$

Usual similarity functions are as follow:

$$
\begin{aligned}
& g_{0}(u, v)=1, \\
& g_{1}(u, v)=\exp \left(-\mu\left(f^{0}(u), f^{0}(v)\right) / \sigma^{2}\right) \text { with } \sigma>0
\end{aligned}
$$

where $\sigma$ depends on the variation of the function $\mu$ and control the similarity scale.

Several choices can be considered for the expression of the feature vectors, depending on the nature of the features to be used for the graph processing. In the context of image processing, one can quote the simplest gray scale or color feature vector $F_{u}$, or the patch feature vector $F_{u}^{\tau}=\bigcup_{v \in \mathcal{W}^{\tau}(u)} F_{v}$ (i.e, the set of values $F_{v}$ where $v$ is in a square window $\mathcal{W}^{\tau}(u)$ of size $(2 \tau+1) \times(2 \tau+1)$ centered at a vertex pixel $u)$, in order to incorporate non-local features.

\section{B. Restoration and Simplification}

1) Scalar image smoothing: In this paragraph, we illustrate the abilities of the normalized $p$-Laplacian diffusion for filtering real images.

A scalar image of $N$ pixels is considered as a function $f^{0}: \mathbb{Z}^{2} \rightarrow \mathbb{R}$ which defines a mapping from the vertices to gray levels. Figure 1 shows sample results obtained with different graph configurations (local and non-local), built from the original noisy image, and different values of parameters $\alpha$ and $\beta$. The first row shows results with $\alpha=1$ and $\beta=0$, what corresponds to the infinity Laplacian. Second row shows results with $\alpha=0$ and $\beta=1$, what corresponds to the normalized Laplacian. Finally third row shows results with $\alpha=0.5$ and $\beta=0.5$ what can be interpreted as an intermediary filtering operator. First and fourth columns present results computed on a usual 4-adjacency grid graph (what is the natural structure of the image), with a constant weight function $w=1$. Second and fifth columns present results obtained on a usual 4-adjacency grid graph, with and image dependent weight function. Finally, third and sixth columns present results computed on a non-local graph, using a $15 \times 15$ neighborhood window and a $5 \times 5$ patch as feature vector.

A second illustration is proposed in Fig. 2, that uses the non-Euclidean triangulated structure of a textured teapot mesh as a graph. Left columns presents results computed using the natural graph structure of the mesh and $w=1$. Finally, right column presents results computed using a largest graph structure, where each vertex $u$ is connected to every vertices $v$ such that there is less than 3 vertices between $u$ and $v$ in the original mesh structure.
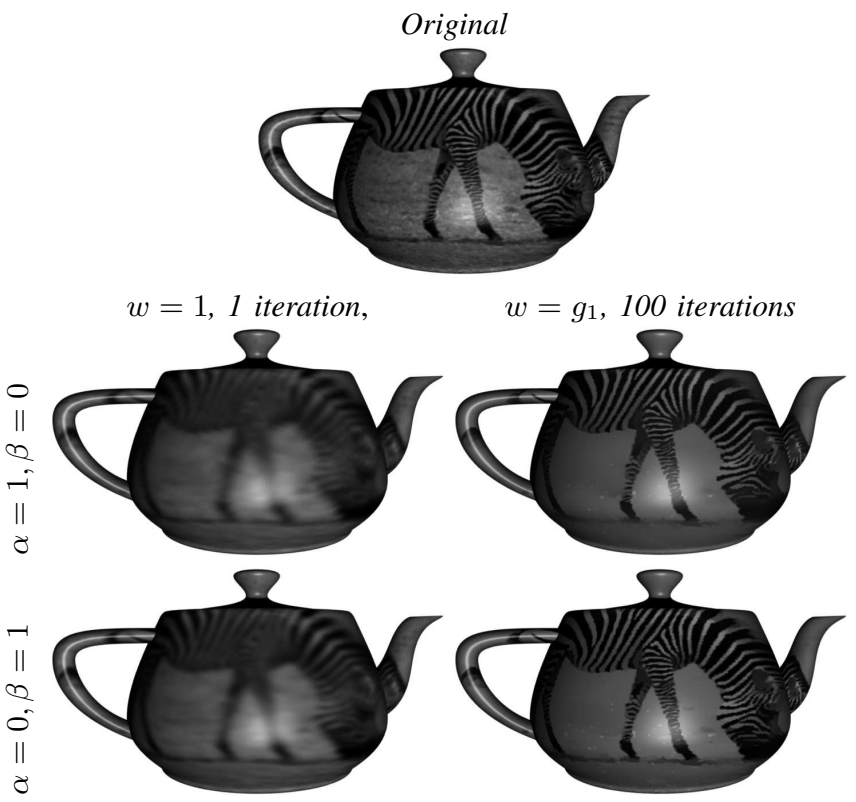

Fig. 2. Textured surface simplification with different parameters values of $\alpha, \beta$ and different weight functions. The graph is local and built from the triangulated mesh structure. First columns shows results using a constant mesh functions. Second column shows results with an adapted weight function. See text for more details.

2) Morphology: In this paragraph, we illustrate morphological $N L E$ and $N L D$ on both image and textured meshes structures. Figure 3 presents results of both morphological processes on a natural image with different graph configurations, weight functions and number of iterations. First and fourth columns show results for a 4 -adjacency grid graph, with a constant weight (respectively 5 an 10 iterations). Second and fifth columns show results for a 4-adjacency grid graph, with image dependent weight (respectively 5 an 10 iterations). Finally, third and sixth columns show non-local graph using $15 \times 15$ neighborhood window and $5 \times 5$ patches (respectively 5 an 10 iterations). Figure 4 presents results of these same processes on a textured mesh, using the natural graph structure of the mesh, with different weight functions (unweighted and data dependent).

\section{Interpolation}

Many tasks in image processing, computer vision and machine learning can be formulated as interpolation problems. Image and video colorization, inpainting and semi-supervised 

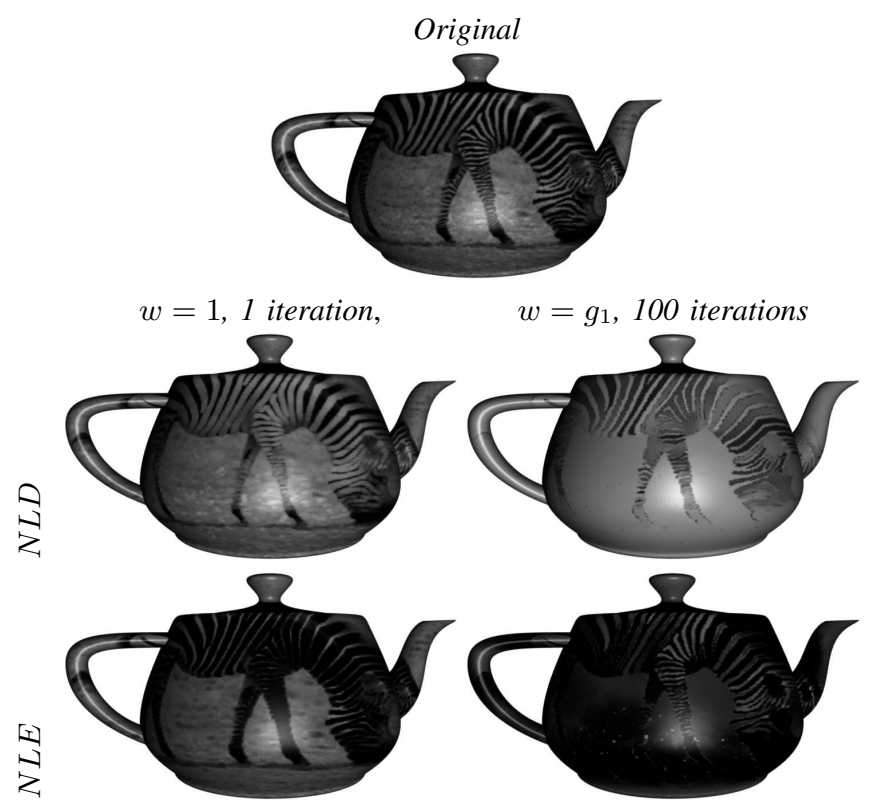

Fig. 4. Non-local morphology ( $N L D$ and $N L E)$ on a textured surface, using different weight functions in local and non-local configurations. The graph is local and built from the triangulated mesh structure. First columns shows results using a constant mesh functions. Second column shows results with an adapted weight function. See text for more details.

segmentation or clustering are examples of these interpolation problems. Interpolating data consists in constructing new values for missing data in coherence with a set of known data. In this paper, we propose to use the non-local normalized $p$ Laplacian as a unified framework for solution for both semisupervised segmentation or clustering and image inpainting. For this, we solve the following Dirichlet problem:

$$
\begin{cases}\Delta_{w, \alpha, \beta}(f)(u)=0 & u \in V_{0} \\ f(u)=g(u) & u \in V-V_{0}=\partial V_{0}\end{cases}
$$

where $V_{0} \subset V$ is the subset of vertices associated to the missing information. The initial value function $g$ is application dependent and will be defined for each application in the sequel.

1) Semi supervised Segmentation and Classification: In the case of image semi supervised segmentation, graph-based approaches have became very popular in recent years. Many graph-based algorithms for image segmentation have been proposed, such as graph-cuts [52], random walker [53], shortestpaths [54], [55],watershed [56], [57], [58] or frameworks that unify some of the previous methods (such as as powerwatershed) [59], [60]. Recently, these algorithms were all placed into a common framework [59] that allows them to be seen as special cases of a single general semi-supervised algorithms. Several popular approaches [29], [54], [55], [58], [61], [62] to perform a graph clustering consist in computing a graph partition from the set of user's seeds and a metric. Interested reader should refer to [61] for more details.

In this paper, we propose to consider this problem as an interpolation problem, where the function to interpolate is the label function. Using equation (76), and considering two classes $A$ and $B$, the initial value label function $g$ is defined as follow

$$
\left\{\begin{array}{l}
g(u)=-1 \text { if } u \in A \\
g(u)=1 \text { if } u \in B \\
g(u)=0 \text { otherwise }
\end{array}\right.
$$

At convergence, the class membership can be easily computed by a simple threshold on the sign of $f$

Remark: In the case of more than two classes, multi-classes segmentation can be performed by several segmentation of one class versus the others.

Natural image segmentation using non-local graph: In this paragraph, we show the benefits of non-local schemes as compared to local ones for semi-supervised image segmentation, especially for images that contain fine and repetitive structures. Figure 5 presents several segmentation results of a natural tiger image, with two different (local and non-local) graph constructions and different values for parameters $\alpha$ and $\beta$. The local graph is built as a usual 4-adjacency grid graph where each pixel is characterized by it's color feature vector. The non-local one is built using a $11 \times 11$ neighborhood window and each pixel is characterized by a $3 \times 3$ patch of color feature vectors.
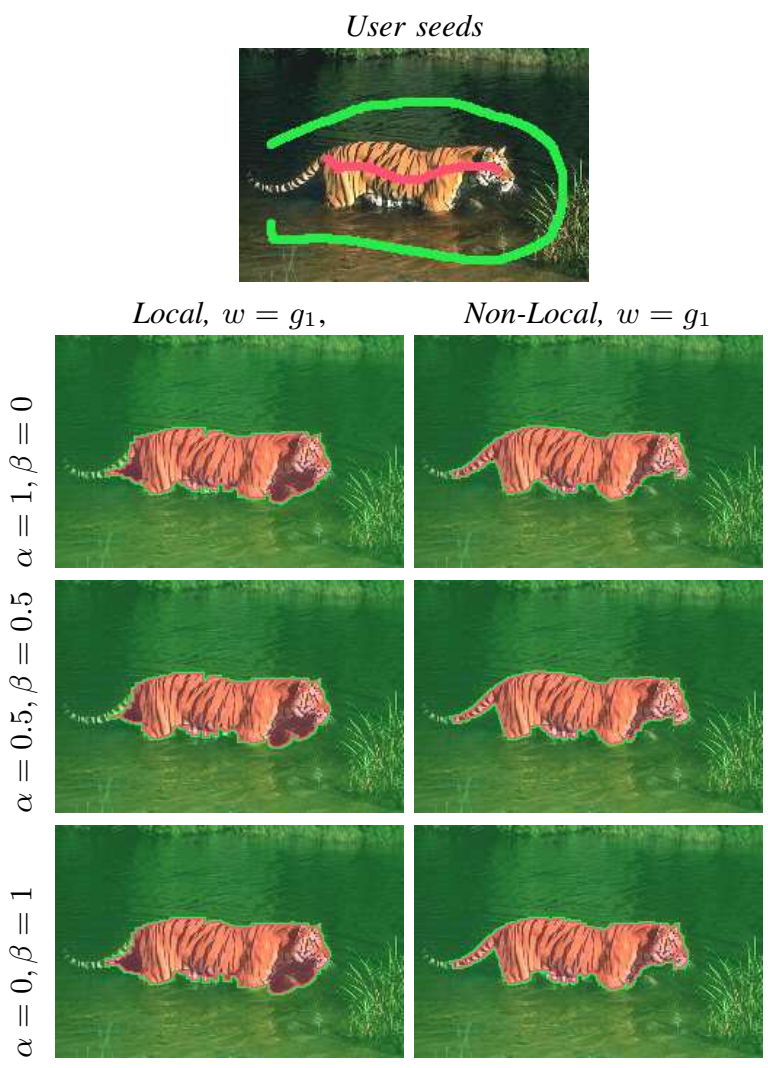

Fig. 5. Semi-supervised natural image segmentation with $\Delta_{w, \alpha, \beta}$. First column presents results with a local 4 -adjacency grid graph where each pixel is characterized by it's color feature vector. Second columns presents nonlocal results obtained with a larger neighborhood (each pixel $u$ is linked with any pixel in a $11 \times 11$ window centered on $u$ ) and pixels are characterized by patches of size $3 \times 3$. In both cases, results are provided for $\alpha=1, \beta=$ $0\left(\Delta_{\infty}\right), \alpha=0.5, \beta=0.5$ and $\alpha=0, \beta=1$. See text for more details. 
Comparison with state-of-the-art methods for semisupervised image segmentation: In this paragraph, we provide (Fig. 6) comparative results for semi-supervised segmentation, between our method and the most competitive methods in the literature, such as graph cut, powerwatershed, random walker, shortest path and watershed. These three images come from the grabcut database and seeds are eroded versions of the ones provided with the database (the seeds and comparative results are reproduced as in Couprie's paper [59]). To demonstrate the performance of our formulation, it is illustrated with the best results beyond all combinations of $\alpha$ and $\beta$ and local or non-local configurations. As it can be seen from the results, our method provides competitive results with all state-of-the-arts methods, with benefits when used on textured images.

Natural image segmentation using $R A G$ representation: In some cases, in particular when the objects to be segmented are numerous and not adjacent, the natural grid-representation of images makes a segmentation impossible (unless building a very huge and complete graph, that slows down a lot the process or hand-labeling every object, what is not an acceptable solution). To overcome this limitation we propose to use a higher-level image representation using a partitioned version of the image (a region map), on which is built a $k$-ERAG. That is what is illustrated in Fig.7. The original image presents a detail of a cytological slide we want to process to extract the nuclei objects (in dark blue). The nuclei are numerous and not adjacent (separated by background or cytoplasm). We consider the nuclei as the first class and the background plus cytoplasm as the second class, what transform this extraction problem in a classification problem. The second image present the image partition (computed using a geometric diffusion algorithm [29]) which boundaries are presented in red. The graph construction is illustrated for a single vertex whose local edges (edges to adjacent regions) are presented in green and non-local edges (edges to $k$ most similar regions in the whole image) are presented in black. Finally, the third image presents the boundaries of the extracted objects. The extraction was performed by label interpolation $\alpha=1, \beta=0$ (i.e. with $\Delta_{\infty}$ ). One can remark that, due to the non-local edges, all the regions of non adjacent cellular objects have been labelized as cellular objects.

Real data clustering: In this paragraph, we present an illustration of label interpolation using equation (76) (we only consider the case $\alpha=1, \beta=0$ ) for real data clustering. The data is a set of 200 digits (zero and one) from the USPS database that we want to cluster in two classes (zeros vs ones). To simplify the graph construction, we consider each digit as a one-dimensional vector of 256 pixels gray values, and the metric between the digits is a simple Euclidean distance. Then, data are represented as a $k \mathrm{nn}$ graph on which the clustering is processed from for user seeds (two per class). Figure 8 presents the graph, with initial seeds and clustering.

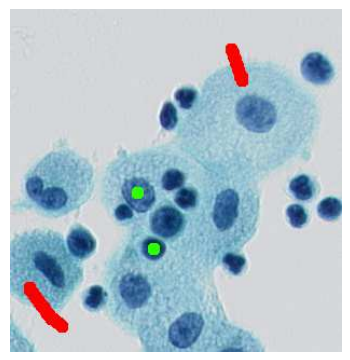

(a)

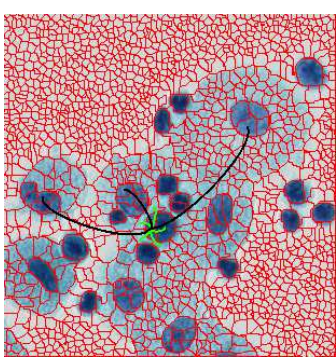

(b)

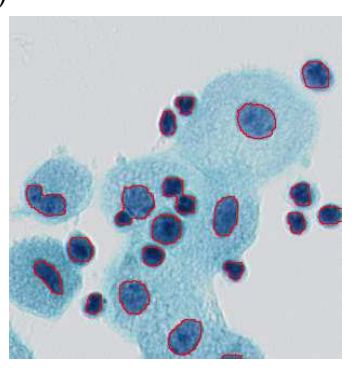

(c)
Fig. 7. Semi supervised natural image segmentation using a partitioned representation of the image : (a) Original image with superimposed two seeds (green and red). (b) Partitioned version of the image (given by the red boundaries). The $k$-ERAG is illustrated for a single vertex with local (green) and non-local (black) edges. (c) Segmentation result (with $\alpha=1, \beta=0\left(\Delta_{\infty}\right)$. See text for more details.
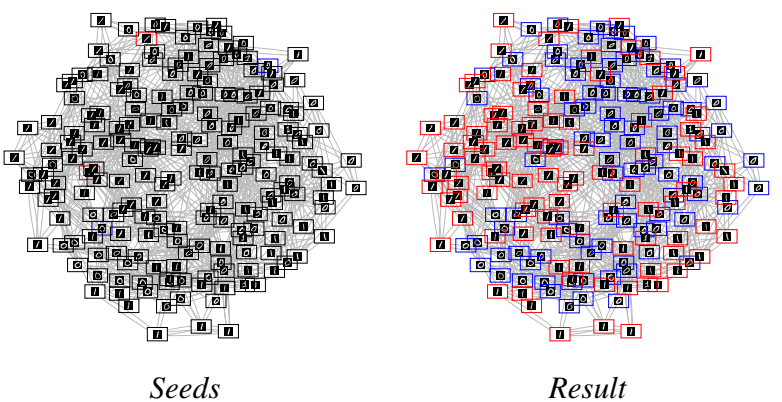

Fig. 8. Semi supervised data clustering with $\Delta_{\alpha, \beta}$. See text for more details.

2) Non-local image inpainting: Digital inpainting is a fundamental problem in image processing and has many applications in different fields. It can be simply resumed as reconstructing a damaged or incomplete image by filling the missing informations in incomplete regions. In recent years, many methods have been developed for interpolating the geometry, the texture or both geometry and texture. Among the methods of interpolation that have been proposed, a number of methods are based on PDEs or variational, see [63], [64] and reference therein. Since the work of Buades et al. on non-local filtering [65], many non-local methods for image inpainting have gained considerable attention these recent years. This is in part due to their superior performance in textured images, known weakness of purely local methods. Recent works tend to unify the local and non-local interpolation approaches [66]. A variational framework for non-local image inpainting has been presented [63]. A discrete non-local regularization framework for image and manifold processing has been proposed. This framework has been used to present a unifying approach of 
local geometric methods and non-local exemplar-based ones for video inpainting [67].

Considering equation (76), inpainting problem can be summarized as follow: $V_{0}$ is the set of pixels with missing information. $g: V \rightarrow \mathcal{H}(V)$ represents the known information. $f: V \rightarrow \mathcal{H}(V)$ represents the image to reconstruct.

This is illustrated in Fig. 9 with different graph configurations and weight functions and different values for parameters $\alpha$ and $\beta$ which correspond to $\Delta_{w, \infty}$ and $\Delta_{w, 2}$. First column presents results for a 4-adjacency grid graph with data dependent weight function. Second column presents results for a nonlocal graph construction using a $31 \times 31$ neighborhood window and $15 \times 15$ patches, with data dependent weight function. Figure 10 gives another inpainting illustration on a natural image, involving more complex structures and textures. The graph is a non-local graph $(25 \times 25$ neighborhood window and $15 \times 15$ patches) with $\alpha=1$ and $\beta=0$.

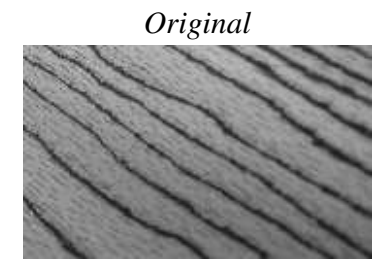

Local, $w=g_{1}$

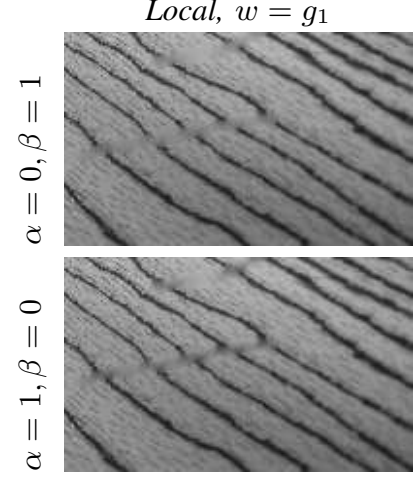

Missing information

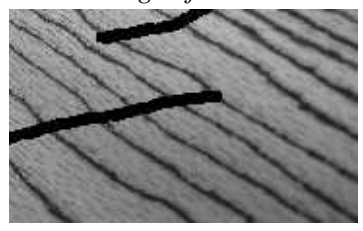

Non Local, $w=g_{1}$

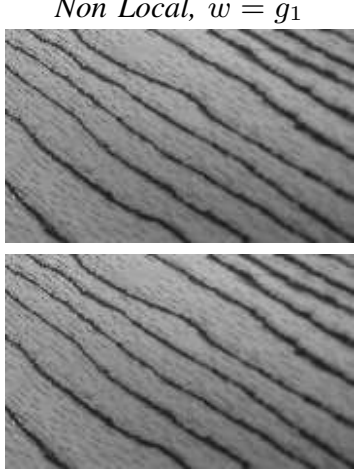

Fig. 9. Natural image inpainting, with different parameters values of $\alpha, \beta$ in local and non-local configurations. Left column presents results with a local 4-adjacency grid graph. Right column presents non-local results, using a $31 \times 31$ neighborhood window and $15 \times 15$ patches.

\section{CONCLUSION}

In this paper, we have introduced a new class of non-local $p$ Laplacian operators as a discrete adaptation of values function of certain random tug-of-war-games. This new class is based on new connections between non-local morphological PDEs, $p$-Laplacian equation and non-local average filtering we have also presented. We have proved existence and uniqueness of the Dirichlet problem involving operators of this new class. Finally, we have illustrated the interest and behavior of such operators in some inverse problems in image processing and machine learning.

\section{REFERENCES}

[1] F. Andreu-Vaillo, J. M. Mazón, J. D. Rossi, and J. J. Toledo-Melero, NonLocal Diffusion Problems. Amer. Math.Soc., 2010.

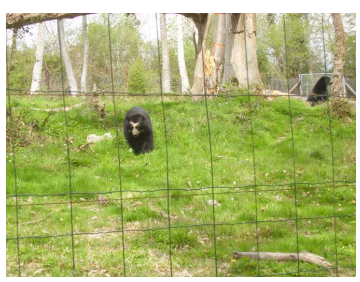

(a)

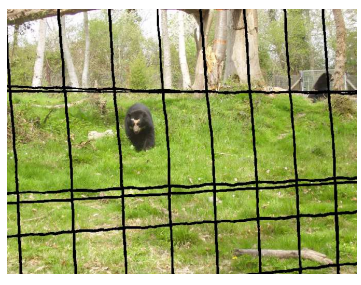

(b)

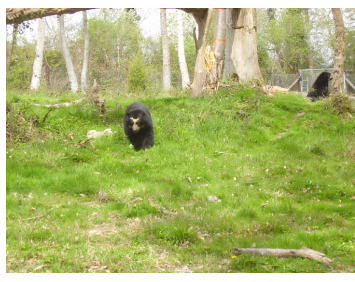

(c)
Fig. 10. Illustration of natural image inpainting using non-local graph configuration, using a $25 \times 25$ window and $5 \times 5$ patches. (a) Original image. (b) Missing information (black). (c) Image reconstruction.

[2] T. Bühler and M. Hein, "Spectral clustering based on the graph plaplacian," in Proceedings of the 26th Annual International Conference on Machine Learning, 2009, pp. 81-88.

[3] M. Hein and T. Bühler, "An inverse power method for nonlinear eigenproblems with applications in 1-spectral clustering and sparse pca," in NIPS, 2010, pp. 847-855.

[4] A. Elmoataz, O. Lézoray, and S. Bougleux, "Nonlocal discrete regularization on weighted graphs: A framework for image and manifold processing," IEEE Trans. Image Process., vol. 17, no. 7, pp. 1047-1060, 2008.

[5] Y. Peres, O. Schramm, S. Sheffield, and D. B. Wilson, "Tug-of-war and the infinity laplacian," J. Amer. Math. Soc., vol. 22, pp. 167-210, 2009.

[6] J. J. Manfredi, M. Parviainen, and J. D. Rossi, "On the definition and properties of p-harmonious functions," To appear in Ann. Sc. Norm. Super. Pisa Cl. Sci.

[7] — , "Dynamic programming principle for tug-of-war games with noise," To appear in ESAIM Control Optim Calc. Var.,

[8] R. R. Coifman and M. Maggioni, "Diffusion wavelets," Appl. Comput. Harmon. Anal., vol. 21, no. 1, pp. 53-94, 2006b.

[9] P. J. O. Maarten H. Jansen, Second Generation Wavelets and Applications. Springer, 2005.

[10] D. K. Hammond, P. Vandergheynst, and R. Gribonval, "Wavelets on graphs via spectral graph theory," Appl. Comput. Harmon. Anal., vol. 30, no. 2, pp. 129-150, 2011.

[11] W. Schwalm, B. Moritz, M. Giota, and M. Schwalm, "Vector difference calculus for physical lattice models," Phy. Rev., vol. 59, pp. 1217-1233, 1999.

[12] P. McDonald and R. Meyers, "Nonlinear elliptic partial difference equations on graphs," Trans. Amer. Math. Soc., vol. 354, no. 12, pp. 5111-5136, 2002.

[13] E. Bendito, A. Carmona, and A. Encinas, "Difference schemes on uniform grids performed by general discrete operators," Appl. Num. Math., vol. 50, pp. 343-370, 2004.

[14] L. Grady and J. R. Polimeni, Discrete Calculus: Applied Analysis on Graphs for Computational Science. Springer, 2010.

[15] R. Courant, K. Friedrichs, and H. Lewy, "On the partial difference equations of mathematical physics," Math. Ann., vol. 100, pp. 32-74, 1928.

[16] A. Bensoussan and J.-L. Menaldi, "Difference equations on weighted graphs," Convex Anal., vol. 12, no. 1, pp. 13-44, 2003.

[17] J. M. Neuberger, "Nonlinear elliptic partial difference equations on graphs," Exp. Math., vol. 15, no. 1, pp. 91-107, 2006.

[18] J.-H. Park and S.-Y. Chung, "Positive solutions for discrete boundary value problems involving the p-laplacian with potential terms," Comput. Math. Appl., vol. 61, pp. 17-29, 2011.

[19] Y.-S. Lee and S.-Y. Chung, "Extinction and positivity of solutions of the p-laplacian evolution equation on networks," J. Math. Anal. Appl., 2011.

[20] L. I. Rudin, S. Osher, and E. Fatemi, "Nonlinear total variation based noise removal algorithms," Phys. D, vol. 60, pp. 259-268, 1992. 
[21] S. Osher and J. Shen, "Digitized pde method for data restoration," Anal. Comput. Methods. Appl. Math., pp. 751-771, 2000.

[22] T. F. Chan, S. Osher, and J. Shen, "The digital tv filter and nonlinear denoising," IEEE Trans. Image Process., vol. 10, pp. 231-241, 2001.

[23] D. Zhou and B. Schölkopf, Discrete Regularization, ser. Adaptive computation and machine learning, 2006, ch. 3.13, pp. 221-232.

[24] S. Bougleux, A. Elmoataz, and M. Melkemi, "Discrete regularization on weighted graphs for image and mesh filtering," in Proc. SSVM, 2007, pp. $128-139$.

[25] O. Lezoray, A. Elmoataz, and S. Bougleux, "Graph regularization for color image processing," Computer Vision and Image Understanding (CVIU), vol. 107, no. 1-2, pp. 38-55, 2007.

[26] G. Gilboa and S. Oser, "Nonlocal operators with applications to image processing," Multiscale Model. Simul., vol. 7, pp. 1005-1028, 2008.

[27] L. Caffarelli, "Non-local diffusions, drifts and games," in Nonlinear Partial Differential Equations. Springer Berlin Heidelberg, 2012, vol. 7, pp. 37-52

[28] V.-T. Ta, A. Elmoataz, and O. Lézoray, "Partial difference equations over graphs: Morphological processing of arbitrary discrete data," in Proc. ECCV, ser. LNCS 5304, 2008, pp. 668-680.

[29] X. Desquesnes, A. Elmoataz, O. Lézoray, and V.-T. Ta, "Efficient algorithms for image and high dimensional data processing using eikonal equation on graphs," in International Symposium on Visual Computing, vol. 2, 2010, pp. 647-658.

[30] V. Ta, A. Elmoataz, and O. Lézoray, "Nonlocal pdes-based morphology on weighted graphs for image and data processing," IEEE transactions on Image Processing, vol. 20, no. 6, pp. 1504-1516, 2011.

[31] S. Bougleux, A. Elmoataz, and M. Melkemi, "Local and nonlocal discrete regularization on weighted graphs for image and mesh processing," International Journal of Computer Vision, vol. 84, pp. 220-236, 2009.

[32] A. Elmoataz, O. Lezoray, S. Bougleux, and V. Ta, "Unifying local and nonlocal processing with partial difference operators on weighted graphs," in International Workshop on Local and Non-Local Approximation in Image Processing (LNLA), 2008, pp. 11-26.

[33] F. Shih and S. Cheng, "Adaptive mathematical morphology for edge linking," Inf. Sci., vol. 167, pp. 9-21, 2004.

[34] R. Verdú-Monedero, J. Angulo, and J. Serra, "Spatially-invariant anisotropic morphological filters driven by gradient fields," in Proc. of ISMM, 2009, pp. 115-125.

[35] O. Tankyevych, H. Talbot, P. Dokládal, and N. Passat, "Directionadaptive grey-level morphology. application to $3 \mathrm{~d}$ vascular brain imaging," in Proc. ICP, 2009, pp. 2261-2264.

[36] M. Charif-Chefchaouni and D. Schonfeld, "Spatially-variant mathematical morphology: Minimal basis representation," in Proc. of ISMM, 1996, pp. $49-56$.

[37] N. Bouaynaya, M. Charif-Chefchaouni, and D. Schonfeld, "Theoretical foundations of spatially-variant mathematical morphologypart i: Binary images," IEEE Trans. Pattern Anal. Mach. Intell., vol. 30, no. 5, pp. 823-836, 2008

[38] P. Maragos and C.Vachier, "Overviewof adaptive morphology: Trends and perspectives," in Proc. ICIP, 2009, pp. 2241-2244.

[39] R. Lerallut, E. Decencire, and F. Meyer, "Image filtering using morphological amoebas," Image Vis. Comput., vol. 25, pp. 395-404, 2007.

[40] L. Vincent, "Graphs and mathematical morphology," Signal Processing, no. 16 , pp. 365-388, 1989.

[41] L. V. H. Heijmans, "Graph morphology in image analysis," Mathematical Morphology in Image Processing, pp. 171-203, 1992

[42] J. A. F. Meyer, "Micro-viscous morphological operators," Mathe- matical Morphology and its Application to Signal and Image Processing, pp. 165-176, 2007

[43] J. S. J. Cousty, L. Najman, "Some morphological operators in graph spaces, mathematical morphology and its application to signal and image processing," Lecture Notes in Computer Science, vol. 5720, pp. 149-160, 2009.

[44] F. M. J. Stawiaski, "Minimum spanning tree adaptive image filtering," in ICIP, 2009, pp. 2245-2248.

[45] G. Sapiro, R. Kimmel, D. Shaked, B. B. Kimia, and A. M. Bruckstein, "Implementing continuous-scale morphology via curve evolution," Pattern Recognit., vol. 26, no. 9, pp. 1363-1372, 1993.

[46] R. W. Brockett and P. Maragos, "Evolution equations for continuousscale morphology," in Proc. ICASSP, vol. 3, 1992, pp. 125-128.

[47] P. Maragos and C. Vachier, "A pde formulation for viscous morphological operators with extensions to intensity-adaptive operators," in ICIP, 2008, pp. 2200-2202.

[48] S. Osher and J. A. Sethian, "Fronts propagating with curvature dependent speed: Algorithms based on hamilton-jacobi formulations," $J$. Comput. Phys., vol. 79, pp. 12-49, 1988.
[49] G. Aronsson, "Extension of functions satisfying lipschitz conditions," Arkiv för Matematik, vol. 6, no. 6, pp. 551-561, 1967.

[50] D. Kawohl, "Variations on the p-laplacian," Cont. Math., no. 540, pp. $35-46,2011$.

[51] A. Chambolle, E. Lindgren, and R. Monneau, "The hölder infinite laplacian and hölder extensions," Preprint, 2010.

[52] Y. Boykov and M.-P. Jolly, "Interactive graph cuts for optimal boundary \& region segmentation of objects in n-d images," in Proc. ICCV, vol. 1 , 2001, pp. 105-112.

[53] L. Grady, "Random walks for image segmentation," IEEE Trans. Pattern Anal. Mach. Intell., vol. 28, pp. 1768-1783, 2006.

[54] X. Bai and G. Sapiro, "Geodesic matting: A framework for fast interactive image and video segmentation and matting," Int. J. Comput. Vis., vol. 82, pp. 113-132, 2009.

[55] A. X. Falcão, J. Stolfi, and R. de Alencar Lotufo, "The image foresting transform: Theory, algorithms, and applications," IEEE Trans. Pattern Anal. Mach. Intell., vol. 26, pp. 19-29, 2004.

[56] L. Vincent and P. Soille, "Watersheds in digital spaces: An efficient algorithm based on immersion simulations," IEEE Trans. Pattern Anal. Mach. Intel., no. 16, pp. 583-598, 1991.

[57] G. Bertrand, "On topological watersheds." J. Math. Imaging Vis., vol. 2-3, no. 22, pp. 217-230, 2005.

[58] J. Cousty, G. Bertrand, L. Najman, and M. Couprie, "Watershed cuts: Minimum spanning forests and the drop of water principle," IEEE Trans. Pattern Anal. Mach. Intel., vol. 31, no. 8, pp. 1362-1374, 2009.

[59] C. Couprie, L. Grady, L. Najman, and H. Talbot, "Power watersheds: A unifying graph-based optimization framework," IEEE Trans. Pattern Anal. Mach. Intell., vol. 33, no. 7, pp. 1384-1399, 2011.

[60] A. K. Sinop and L. Grady, "A seeded image segmentation framework unifying graph cuts and random walker which yields a new algorithm," in Proc. ICCV, 2007, pp. 1-8.

[61] X. Desquesnes, A. Elmoataz, O. Lézoray, and V.-T. Ta, "Eikonal equation adaptation on weighted graphs: fast geometric diffusion process for local and non-local image and data processing," Submitted, 2011.

[62] F. Meyer, "Topographic distance and watershed lines," Signal Proc., vol. 38, pp. 119-125, 1994.

[63] P. Arias, G. Facciolo, V. Caselles, and G. Sapiro, "A variational framework for exemplar-based image inpainting," Int. J. of Comp. Vis., vol. 93, no. 3, pp. 319-347, 2011

[64] C.-B. Schoenlieb and A. Bertozzi, "Unconditionally stable schemes for higher order inpainting," Comm. Math. Sci., vol. 2, no. 9, pp. 413-457, 2011.

[65] A. Buades, B. Coll, and J.-M. Morel, "Nonlocal image and movie denoising," Int. J. Comput. Vis., vol. 76, pp. 123-139, 2008.

[66] G. Gilboa and S. Osher, "Nonlocal linear image regularization and supervised segmentation," Multiscale Model. Simul., vol. 2, no. 6, pp. 595-630, 2007

[67] M. Ghoniem, A. Elmoataz, and O. Lézoray, "Discrete infinity harmonic functions: towards a unified interpolation framework on graphs," in Int. Conf. on Image Process. (IEEE), 2011.

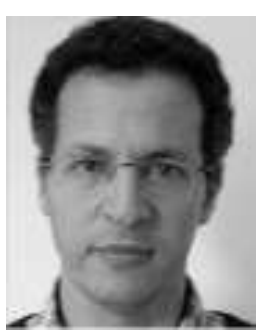

Abderrahim Elmoataz is a full-time Professor of computer science in the Computer Science Department, Université de Caen Basse-Normandie, France. His research concerns PDEs on graphs with applications in image processing and machine learning. 


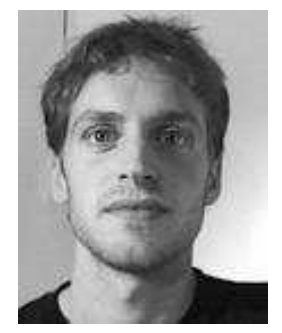

Xavier Desquesnes received the M.Sc. degree in computer science from the Universit de Caen BasseNormandie, France in 2009. Since 2009 he was a $\mathrm{PhD}$ student in computer science and Assistant Professor in computer science with the School of Engineers of Caen (ENSICAEN), France. His research mainly concerns image and data processing with graph-based fronts propagation and filtering techniques.

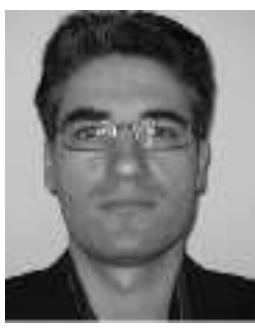

Olivier Lézoray received the M.Sc. and Doctoral degrees in computer science from the Université de Caen Basse-Normandie, France, in 1996 and 2000, respectively. From 1999 to 2000, he was an Assistant Professor with the Computer Science Department, Université de Caen Basse-Normandie. From 2000 to 2009, he was an Associate Professor Communications, Networks and Services Department, Cherbourg Institute of Technology. Since 2010, he has been a Full Professor at the Cherbourg Institute of Technology. His research concerns color image segmentation and filtering (graph-based variational and morphological methods) and machine learning techniques for image mining (neural networks and support vector machines). 

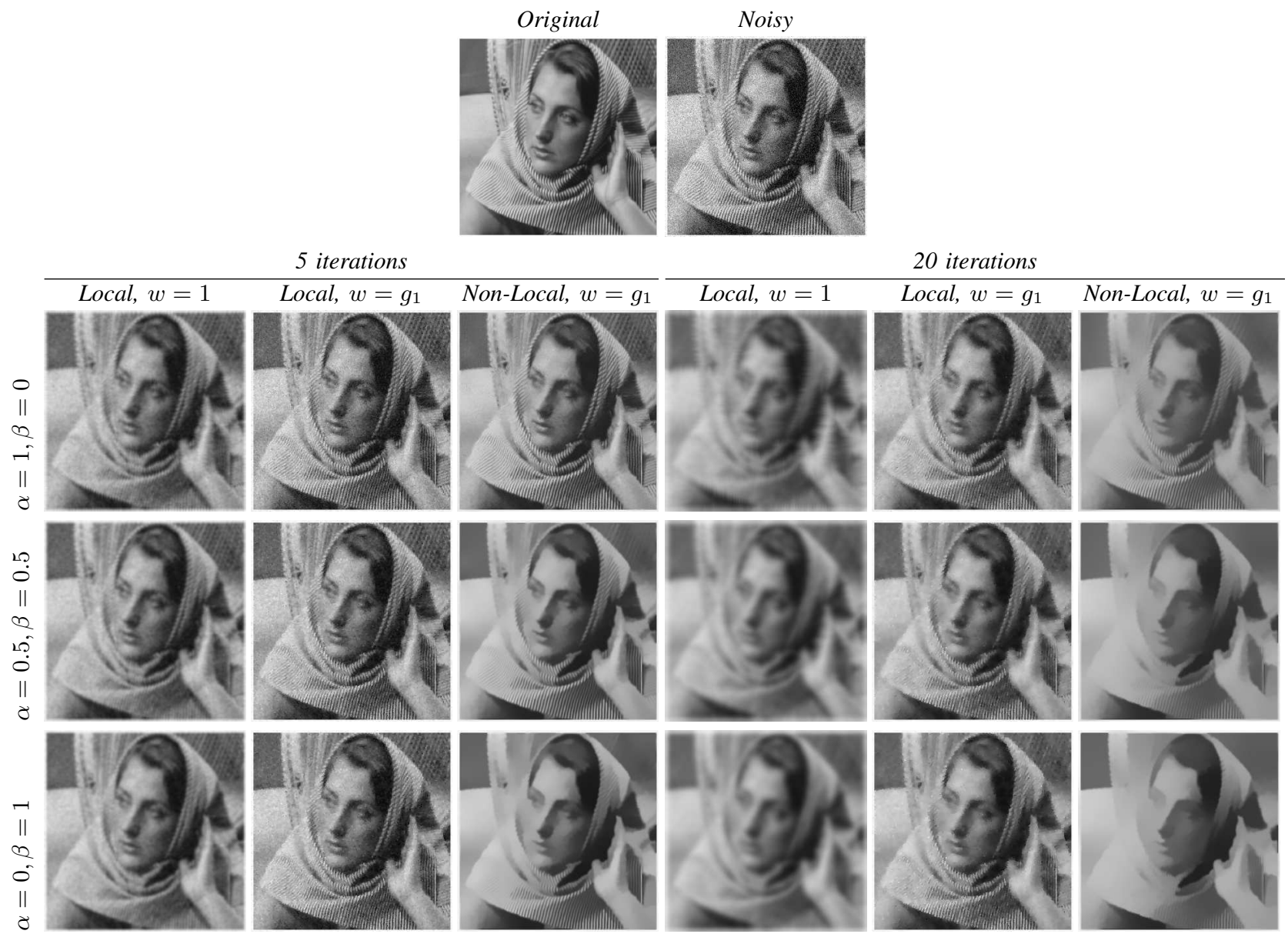

Fig. 1. Scalar image simplification on a gray level image with different parameters values of $\alpha, \beta$ and different weight functions in local and non-local configurations, with 5 or 20 iterations. First row shows results for $\alpha=1, \beta=0\left(\Delta_{\infty}\right)$. Second row shows results for $\alpha=0.5, \beta=0.5$. Finally, third column shows results for $\alpha=0, \beta=1\left(\Delta_{2}\right)$. See text for more details.
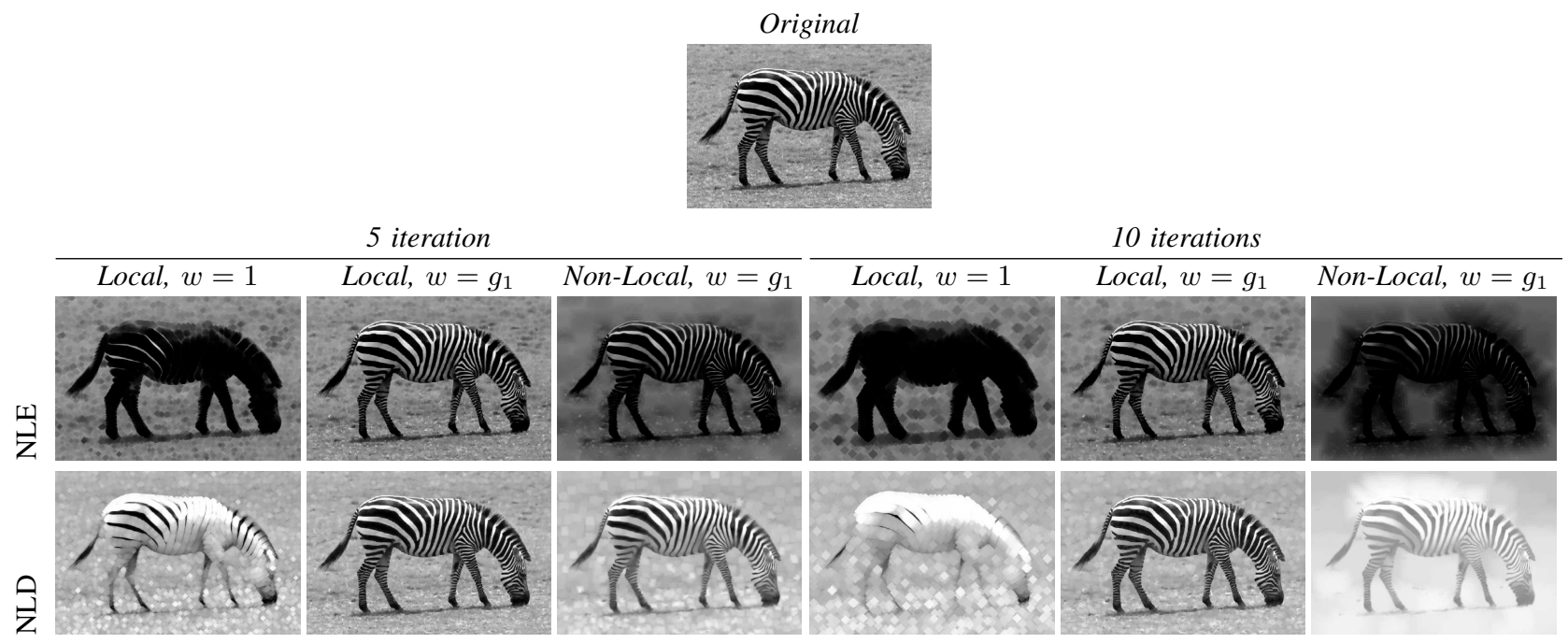

Fig. 3. Illustration of non-local morphological erosion and dilation. First and fourth columns show results for a 4-adjacency grid graph, with a constant weight (respectively 5 an 10 iterations). Second and fifth columns show results for a 4 -adjacency grid graph, with image depend-ant weight (respectively 5 an 10 iterations). Finally, third and sixth columns show non-local graph using $15 \times 15$ neighborhood window and $5 \times 5$ patches (respectively 5 an 10 iterations). See text for more details. 

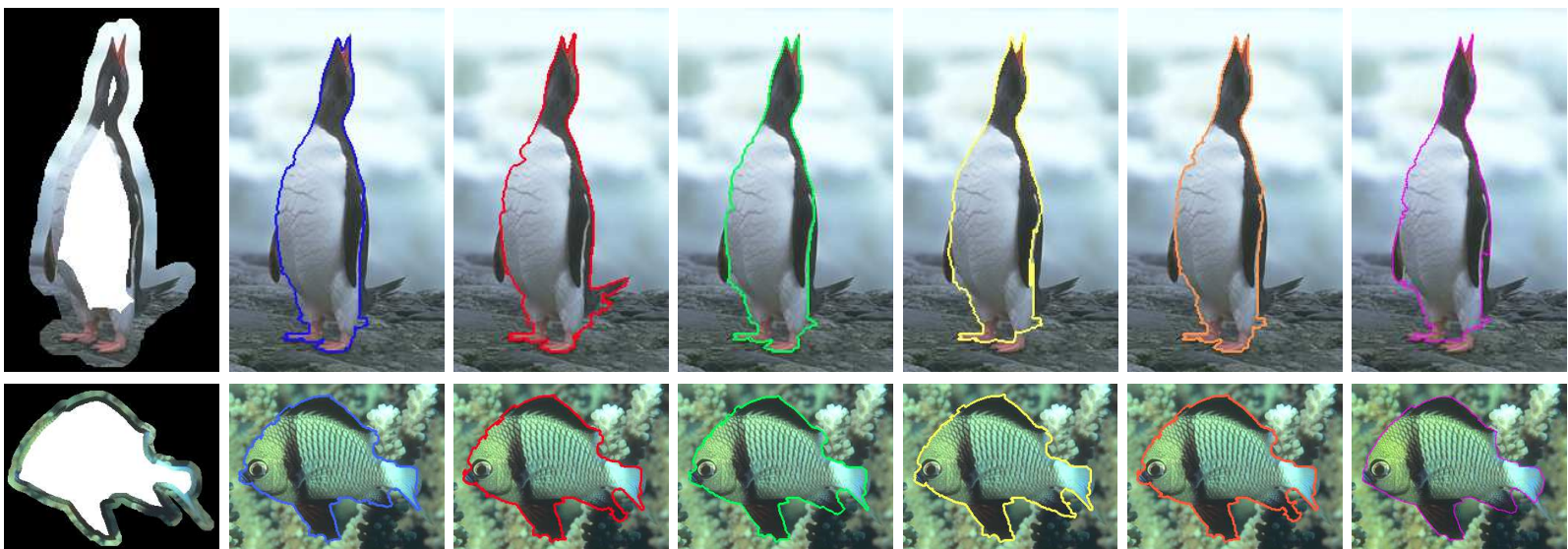

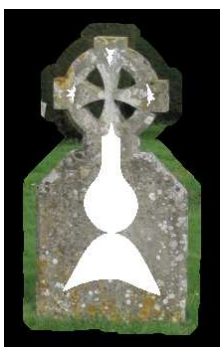

Seeds

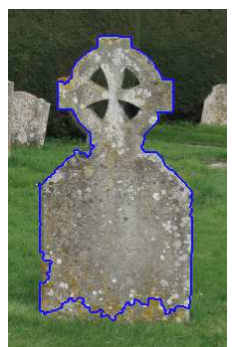

Graph-cut

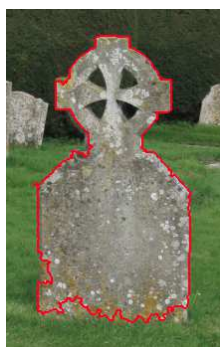

PowerWatershed

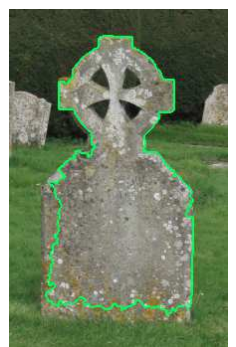

Random Walker

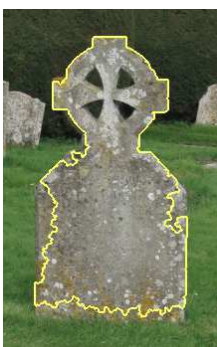

Shortest Path

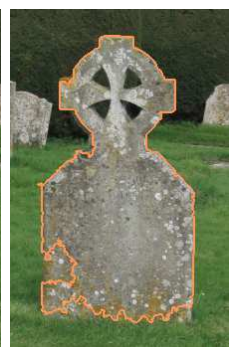

WaterShed

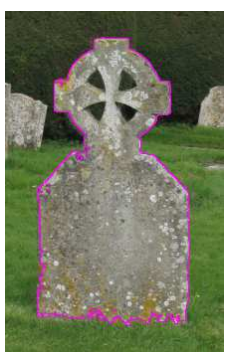

Our

Fig. 6. Comparison between our approach and other local methods for semi-supervised image segmentation. Images come from the grabcut database and seeds are eroded versions of ones provided in the database. For each image, we provide the best result beyond all combinations of $\alpha$ and $\beta$ and local or non-local configurations. 